\title{
Die zukünftige Entwicklung der Grundschulversorgung im Kontext ausgewählter Wanderungsszenarien
}

\section{Eine kleinräumige Mikrosimulationsstudie am Beispiel der Stadt Trier}

\author{
Sebastian Dräger (D) · Johannes Kopp • Ralf Münnich • Simon Schmaus
}

Eingegangen: 16. August 2021 / Angenommen: 25. Januar 2022 / Online publiziert: 17. Februar 2022

(C) Der/die Autor(en) 2022

Zusammenfassung Um die Potentiale der Mikrosimulation als ein Instrument zur Analyse zukünftiger Versorgungslagen in verschiedenen (sozialpolitischen) Bereichen aufzuzeigen, wurde am Beispiel der Stadt Trier eine Mikrosimulationsstudie durchgeführt, bei der der Einfluss unterschiedlicher Wanderungsszenarien auf die Situation der Grundschülerinnen und Grundschüler im Stadtgebiet untersucht wurde. Die Datenbasis stellt eine synthetische Grundgesamtheit der Bevölkerung auf Basis der Zensusergebnisse 2011 dar, welche mit Hilfe von Zensusgitterzellen geografisch im Stadtgebiet verortet und modellbasiert in die Zukunft fortgeschrieben wurde. Außerdem liegen für Trier für die Jahre 2011 bis 2018 präzise Zahlen zu Schülerinnen und Schülern der Elementarstufe vor, an denen nicht nur die Simulationsgesamtheit konfiguriert werden kann, sondern die auch als zentrale Benchmarks für die Validität der Fortschreibungsmodule und -Parameter dienen können.

Schlüsselwörter Grundschulinfrastruktur · Bedarfsplanung · Demographie · Kleinräumige Mikrosimulationsmodelle $\cdot$ Georeferenzierung

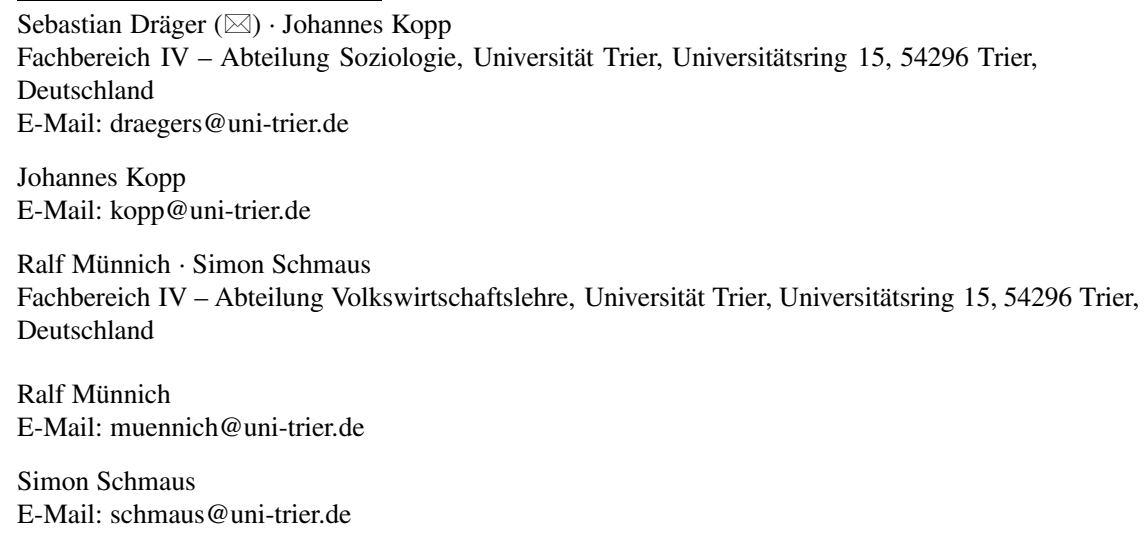




\title{
The future development of primary school demand in the context of selected migration scenarios
}

A spatial microsimulation study using the city of Trier as an example

\begin{abstract}
In order to demonstrate the potential of microsimulation as an instrument for analysing future supply situations in various (socio-political) areas, a microsimulation study was carried out using the city of Trier as an example, in which the influence of different migration scenarios on the situation of primary school pupils in the urban area was investigated. The data basis is a synthetic population based on the census results of 2011, which was geographically located in the city area with the help of census grid cells and was extrapolated into the future based on models. In addition, precise figures on elementary school students are available for Trier for the years 2011 to 2018, which can not only be used to configure the simulation population, but can also serve as central benchmarks for the validity of the update modules and parameters.
\end{abstract}

Keywords Primary school infrastructure - Demand planning - Demographics · Spatial microsimulation models $\cdot$ Georeferencing

\section{Einleitung}

Eine kürzlich von der Bertelsmann Stiftung veröffentlichte Studie zur zukünftigen Schülerzahl im Primarbereich (Klemm und Zorn 2019) hat ergeben, dass die von der Kultusministerkonferenz (KMK) antizipierten Zahlen die Realität deutlich zu unterschätzen scheinen. Während im Mai 2018 seitens der Verantwortlichen bis zum Schuljahr 2025/2026 ein Anstieg um 5,7 Prozent erwartet wurde (KMK Kultusministekonferenz 2018), wies bereits im Juni 2019 die Bevölkerungsvorausberechnung durch das Statistische Bundesamt einen Zuwachs um 11,8 Prozent aus - und zwar in der Variante, die den geringsten Anstieg der Sechs- bis unter Zehnjährigen annimmt (Statistisches Bundesamt 2019). In Zahlen bedeutet das eine Differenz von rund 168.000 Kinder im entsprechenden Alter, die bis zum Jahr 2025 zusätzlich beschult werden müssen und infolgedessen eben auch einen Mehrbedarf an ohnehin schon knappen Lehrkräften im Primarbereich. Die Planung solcher zukünftigen Bedarfe, nicht nur an Personal, sondern auch beispielsweise an Einrichtungen, aber auch die politischen und praktischen Reaktionen benötigen dabei aber eine gewisse Vorlaufzeit, aufgrund von oftmals nicht unerheblichen Ausbildungszeiten oder wegen langwieriger bürokratischer und planerischer Prozesse. Daher sind verlässliche Zahlen und Aussagen über zukünftige Entwicklungen unerlässlich, um eine effiziente Planung von Interventionen und Investitionen durchführen zu können. Neben den Bevölkerungsvorausberechnungen der statistischen Ämter stellen sogenannte Simulationsmodelle ein relativ neues methodisches Instrument der sozialwissenschaftlichen und demographischen Forschung dar, um solche zukünftigen Umstände nicht bloß intuitiv annehmen zu müssen, sondern auch objektiv und empirisch begründen zu können. Dabei handelt es sich um Modelle, die - im Falle von Mikrosimulationsmodellen - auf Basis des antizipierten Verhaltens von Einheiten auf 
der Individualebene Entwicklungen auf der Makroebene prognostizieren sollen. Die Erklärung makrostruktureller Veränderungen - wie beispielsweise die Entwicklung der Grundschülerzahl - durch die Modellierung der Prozesse auf der Mikroebene erlaubt es darüber hinaus zu testen, wie sich bestimmte Szenarien auf der Makrooder auf der Mikroebene auf die zukünftigen Begebenheiten auswirken. Ziel dieses Beitrags ist es nun, am Beispiel der Stadt Trier exemplarisch die Anwendung solcher Mikrosimulationsmodelle für die Generierung valider Informationen über zukünftige Entwicklungen im Bereich der Grundschulen und vor allem der Zahl der Schülerinnen und Schüler zu demonstrieren. Neben der Fokussierung auf die Mikroebene, die es in Abgrenzung zu den gängigen Bevölkerungsvorausberechnungen zusätzlich erlaubt, in den Modellen und Projektionen die Interdependenzen von verschiedenen Ereignissen - ob als Wechselwirkung biographischer Ereignisse oder als (fiktive) Szenarien - und ihre Wirkung auf die Makroebene darzustellen (Hannappel und Troitzsch 2015), nimmt das dieser Arbeit zugrundeliegende Mikrosimulationsmodell zusätzlich eine regionale, kleinräumige Perspektive ein. Mit Blick auf eine möglichst effiziente Bedarfsplanung ist es nicht nur wichtig zu wissen, wie viele Grundschülerinnen und Grundschüler zukünftig in Trier zu finden sein werden, sondern es ist auch interessant zu wissen, ob es Stadtteile oder Regionen im Stadtgebiet geben wird, in denen die Konzentration von Sechs- bis unter Zehnjährigen Kindern zukünftig besonders hoch sein wird.

Da es sich bei dieser Kombination aus Georeferenzierung von Daten und Mikrosimulation um die Verknüpfung von zwei noch eher neuen und in der Praxis seltener angewendeten Methoden handelt, müssen in Kap. 2 dieses Beitrags einige methodische Grundlagen diskutiert werden. Hierzu gehört neben der Erstellung und der räumlichen Verortung einer geeigneten Datenbasis auch eine Auseinandersetzung mit den für Mikrosimulationsmodelle spezifischen Fortschreibungsmodulen und -modellen. Kap. 3 widmet sich dann der Frage der Qualität und der Validität der Daten und der Fortschreibungsroutinen, indem ausgehend vom Startjahr 2011 die simulierten Ergebnisse mit den Informationen über die tatsächliche Anzahl an Grundschülerinnen und Grundschülern bis zum Jahr 2018 verglichen werden. Darauf aufbauend werden hier auch einige Simulationsergebnisse unter verschiedenen Wanderungsszenarien bis ins Jahr 2061 vorgestellt und gezeigt, wie sich diese auch auf die räumliche Verortung auswirken. Kap. 4 schließt den Artikel mit einer Zusammenfassung und einem kurzen Ausblick.

\section{Methodisches Vorgehen}

Mikrosimulationen im Allgemeinen beschreiben Techniken, die es erlauben, Entwicklungen von Systemen (Populationen) und Einflüsse politischer Maßnahmen über die Betrachtung der kleinsten enthaltenen Einheiten zu untersuchen. Diese Einheiten können sich je nach Untersuchungsgegenstand unterscheiden. Im Kontext sozialwissenschaftlicher sowie ökonomischer Fragestellungen handelt es sich meist um Haushalte oder Personen. In der Praxis finden sich unzählige Möglichkeiten, Mikrosimulationen umzusetzen. Diese gehen von sogenannten statischen Modellen, in denen nur einzelne politische Maßnahmen wie Steuersysteme angepasst werden, 
bis hin zu deutlich komplexeren dynamischen Modellierungen, die individuelles Verhalten in die Zukunft fortschreiben (Li und O'Donoghue 2013; Burgard et al. 2020a).

Dass Mikrosimulationsmodelle in den Sozialwissenschaften als neu gelten, ist nicht zwangsweise ihrem Alter, sondern viel mehr der enorm gestiegenen Rechenleistung sowie der Verfügbarkeit vieler Daten auf Individualebene geschuldet. Bereits 1957 äußerte Guy H. Orcutt in seinem vielzitierten Aufsatz A New Type of Socio-Economic System (Orcutt 1957) Kritik an den bestehenden Methoden und deren Unfähigkeit individuelles Verhalten von Akteuren auf der Mikroebene zu berücksichtigen und somit auch keine Simulation beispielsweise der Effekte politischer Reformen auf die gesamte Gesellschaft zuzulassen, da vor allem Prozesse auf der Aggregatebene berücksichtigt wurden. Neben der Berücksichtigung der Mechanismen auf der Mikroebene und der dadurch entstehenden Interdependenzen nicht zwischen einzelnen biographischen Ereignissen eines Individuums, sondern auch zwischen der Mikro- und der Makroebene - stellt die Erweiterung der Mikrosimulationsmodelle um die kleinräumige Perspektive einen weiteren deutlichen Mehrwert dar. Der Vorteil, auch kleinste räumliche Einheiten gesondert analysieren zu können, liegt darin, regionale Disparitäten in den Blick nehmen zu können. Oftmals wurden lediglich größere Einheiten wie etwa Ost- und Westdeutschland, oder auch die Bundesländer, als Analyseebene herangezogen und verglichen. Allerdings handelt es sich dabei um eine sehr große und oftmals auch heterogene Gliederungsebene, die tiefergehende Unterschiede - etwa zwischen Stadt und Land oder sozial gänzlich unterschiedlichen Milieus - nicht abbilden kann. ${ }^{1}$ Im Falle der Grundschulversorgung besteht aber durchaus sogar Interesse an Entwicklungen in einzelnen Stadtteilen oder gar Straßenzügen, welche sich jedoch erst mit einer sehr kleinteiligen Betrachtung analysieren lassen. Am Beispiel von Trier soll nun gezeigt werden, wie man die entsprechenden Daten auswerten, in ein Simulationsmodell integrieren, in die Zukunft fortschreiben und die Ergebnisse entsprechend einer spezifischen Fragestellung analysieren kann.

\subsection{Basispopulation}

Die Grundlage jeder Mikrosimulation ist ein geeigneter Ausgangsdatensatz, der das zu simulierende System mit den enthaltenen Mikroeinheiten zum Startzeitpunkt repräsentiert. In der Praxis sozialwissenschaftlicher und ökonomischer Simulationen kommen in den meisten Fällen Datensätze aus statistischen Erhebungen zum Einsatz (Li 2011; Li und O'Donoghue 2013). Der Grund liegt neben der leichten Verfügbarkeit insbesondere am großen Umfang enthaltener Informationen. Der Hauptnachteil dieser Daten liegt jedoch in geringen Stichprobenumfängen, die regionalisierte Untersuchungen, wenn überhaupt, nur auf sehr grober Ebene zulassen.

Bei regionalisierten Analysen ist es kaum möglich, einen Sekundärdatensatz zu finden, der nicht nur repräsentativ für die zu untersuchende Grundgesamtheit ist, son-

\footnotetext{
1 Thematisiert wird diese Notwendigkeit der Betrachtung regionaler Disparitäten im Kontext von Mikroanalysen oder -simulationen unter anderem von Chin und Harding (2006) beziehungsweise Tanton (2014) und Tanton (2018).
} 
dern auch alle für die Analyse benötigten (Individual-)Merkmale enthält, vor allem, wenn zusätzlich hohe Anforderungen an die räumliche Differenzierbarkeit gestellt werden. In diesem Fall besteht die Möglichkeit, auf die Erstellung eines synthetischen Datensatzes zurückzugreifen. Hierbei wird anhand vorhandener Informationen und Surveydaten eine Basispopulation erstellt, die zwar nicht real existierenden Individuen entspricht, aber dennoch in dem Sinne realitätsnah ist, dass man mit einer hohen Sicherheit davon ausgehen kann, dass die Strukturen auf den Aggregatebenen den Ergebnissen tatsächlicher Erhebungen gleichkommen, während individuelle Verteilungen aus erhobenen Individualdaten approximiert werden.

Aufgrund der hohen Flexibilität wurde als Basispopulation für diese Arbeit eine realitätsnahe synthetische Grundgesamtheit von Trier mit insgesamt 105.000 Individuen in 50.000 Haushalten erstellt. Die Erstellung dieser Datenbasis erfolgte dabei durch die Erweiterung und Aktualisierung einer Simulationsgesamtheit, die im Rahmen eines Projektes zum Zensus 2011 erstellt wurde (Münnich et al. 2012; Burgard et al. 2019). Um sicherzustellen, dass diese Basispopulation auch den tatsächlichen Begebenheiten in Trier entspricht, wurde diese an die Zensusergebnisse aus dem Jahr 2011 (Statistische Ämter des Bundes und der Länder 2014) angepasst. Hierzu wurden sowohl Kalibrierungsverfahren (Deville und Särndal 1992) als auch Methoden der heuristischen Optimierung (van Laarhoven und Aarts 1987) verwendet. Der so erstellte Datensatz enthält bereits eine Vielzahl an überwiegend soziodemographischen Merkmalen der synthetischen Individuen. Außerdem besteht die Möglichkeit einen solchen synthetischen Datensatz nach Bedarf um weitere Merkmale der Individuen zu erweitern, sofern dies für neue Fragestellungen oder auch wegen neuer Erkenntnisse über relevante Einflussfaktoren bereits untersuchter Phänomene benötigt wird. Hierzu wird versucht, aus bestehenden Untersuchungen die Parameter für die Verteilung der interessierenden Merkmale bestmöglich zu erfassen, um aus diesen letztlich Vorhersagen für die synthetische Basispopulation treffen zu können. Die Qualität der Modelle lässt sich dann unter dem Kriterium der prädikativen Inferenz, also inwiefern auf diese Weise tatsächlich möglichst realitätsnahe Werte vorhergesagt werden können, beurteilen. ${ }^{2}$ Fügt man auf diese Art weitere Merkmale ein, ist zusätzlich zu berücksichtigen, dass dadurch die neue Datenbasis nicht etwaigen bekannten Verteilungen widerspricht. Hierzu kann man als Benchmarkgrößen oftmals Daten der amtlichen Statistik nutzen, um die modellbasierten Vorhersagen zu kalibrieren und an die Realität anzupassen. Small-Area-Methoden können zusätzlich helfen die Qualität des synthetischen Datensatzes zu verbessern, sofern keine realen und regional differenzierten Vergleichswerte vorhanden sein sollten, an denen man die Basispopulation ausrichten kann. ${ }^{3}$ Für die Analyse der Grundschulversorgung in Trier wurden auf diese Weise allerdings nur wenige Merkmalskombinationen, vornehmlich zu interpersonellen Beziehungsstrukturen, über das Sozio-ökonomische Panel (SOEP - Goebel et al. 2019) und den Mikrozensus (Statistisches Bundesamt 2017) implementiert.

Der Beginn der Schulpflicht und somit der Eintritt in die Grundschule ist nun weitgehend durch die allgemeine Schulpflicht und entsprechende Vorgaben zum

\footnotetext{
2 Zur näheren Beschreibung der Methode siehe Kolb (2013).

${ }^{3}$ Zur Logik der Small-Area-Methoden siehe Münnich et al. (2013).
} 
Eintrittsalter gesetzlich geregelt. ${ }^{4}$ Deshalb genügt es für die hier formulierte Zielsetzung, die Bevölkerungsstruktur unter Berücksichtigung einfacher Fertilitätsmodelle fortzuschreiben, um so kleinräumig die entsprechende Zahl an Schülerinnen und Schüler im Zeitverlauf zu prognostizieren. Die erste Betrachtung des Basisjahres 2011 und der Vergleich der realen mit den in der Simulationsgesamtheit realisierten Zahlen, soll vornehmlich dem Zweck dienen die Realitätsnähe der erstellten synthetischen Basispopulation zu evaluieren. Mit Blick auf die zugrundeliegende Fragestellung ist die zentrale Zielgröße dabei die Zahl der Grundschülerinnen und Grundschüler im Jahre 2011 in Trier. Als Vergleich dienen hierzu die von der Schulbehörde von Trier übermittelten Daten, die - differenziert nach Schule und Klasse - Auskunft über die tatsächliche Zahl der Grundschülerinnen und Grundschüler geben. Demgegenüber enthält die Simulationsgesamtheit allerdings keine genauen Informationen darüber, ob ein Kind eine Grundschule besucht oder nicht. Da es sich bei der Einschulung allerdings um ein Phänomen handelt, welches im Grunde sowohl in Bezug auf die Wahrscheinlichkeit als auch auf den Zeitpunkt des Eintretens determiniert ist, ist es naheliegend, dieses Merkmal über das Alter zu approximieren. Das Schulgesetz von Rheinland-Pfalz sieht vor, dass alle Kinder die bis zum 31. August des entsprechenden Jahres das sechste Lebensjahr vollenden mit Beginn des Schuljahres eingeschult werden (Ministerium für Bildung, Wissenschaft, Weiterbildung und Kultur 2014, §57 des Schulgesetzes von Rheinland-Pfalz). Da eine Grundschullaufbahn in der Regel vier Schuljahre umfasst, wäre somit anzunehmen, dass Zehnjährige bereits nicht mehr zu den Grundschülerinnen oder Grundschülern zu zählen sind. Folgende Grafik zeigt dementsprechend die reale Zahl der Grundschülerinnen und Grundschüler im Vergleich zu der Zahl der Sechs- bis unter Zehnjährigen in der Basispopulation.

Dieser recht basale Vergleich zeigt bereits, dass bei dieser groben Approximation des Grundschulbesuchs die Zahlen der Simulationsgesamtheit die realen Begebenheiten nur leicht überschätzen. Etwas differenzierter kann man diese generelle Übereinstimmung bewerten, wenn man nicht bloß die Gesamtzahlen vergleicht, sondern auch die Verteilung auf die Klassenstufen betrachtet. Analog zur gesamten Grundschülerzahl entspricht den realen Klassenstärken die Zahl der Sechs-, Sieben-, Achtund Neunjährigen. Dieser Vergleich lohnt sich dabei nicht nur mit Blick auf die absoluten Zahlen der beiden Datenquellen, sondern auch bezüglich der Frage, ob die generelle Verteilung auf die Klassenstufen annähernd realistisch ist (Abb. 1).

Bei der Betrachtung der Klassenstufen in den absoluten Zahlen lassen sich leichte Abweichungen erkennen, die in der Summe der Differenz der Gesamtschülerzahl entspricht. Bemerkenswert ist allerdings, dass diese Fehler nicht gleich verteilt sind, sondern gewissermaßen systematisch die erste Klassenstufe in der Approximation über das Alter eher unterschätzt wird, während die späteren Stufen überschätzt werden. Verantwortlich hierfür kann wiederum die Möglichkeit der Einschulung von

\footnotetext{
${ }^{4}$ Es wird hier durchaus davon ausgegangen, dass die Abweichungen von der gesetzlichen Verpflichtung zum Schulbesuch ab dem Alter von sechs Jahren so gering sind, dass diese (zunächst) vernachlässigt werden können. Auch Klassenwiederholungen sind nur in 0,9 Prozent der Fälle überhaupt aufgetreten, weshalb dieser Umstand ebenfalls zu vernachlässigen ist. (Vgl. Statistisches Bundesamt 2018) Ob gegebenenfalls eine Modellierung der Entscheidung wann ein Kind erstmals die Grundschule besucht, vonnöten ist, sollen die Ergebnisse dieser Arbeit zeigen.
} 
Abb. 1 Vergleich der Grundschülerzahlen nach Klassenstufen mit der Basispopulation (2011) (Quelle: Kommunales Bildungsmanagement Trier und eigene Berechnungen)

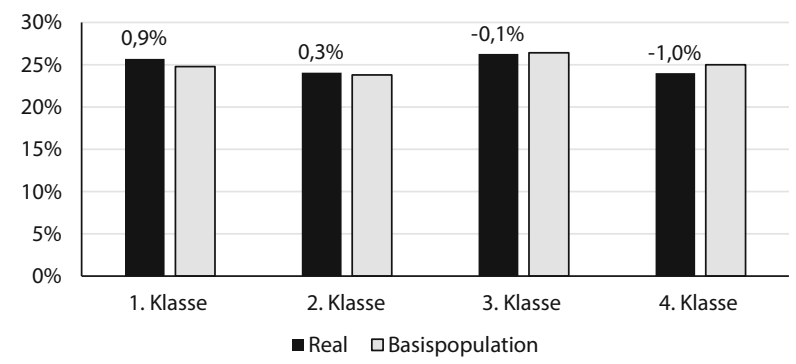

Kindern schon mit fünf Jahren, sein. Dies würde sowohl die Unterschätzung der ersten Klasse - hier werden in der Simulation nur Sechsjährige verortet - als auch die Überschätzung der vierten Klassenstufe erklären, da Kinder die mit fünf Jahren eingeschult wurden bereits mit acht Jahren ihre Grundschullaufbahn beenden. Aber auch neue Phänomene, wie der Wegzug im Laufe des Zeitraums des Grundschulbesuchs, können hier eine Rolle spielen. Diese leichten Differenzen zeigen sich dann auch, wenn man den Anteil an der Gesamtschülerzahl je Klassenstufe betrachtet und somit die Abweichung in der absoluten Gesamtgrundschülerzahl nicht berücksichtigt. Alle Darstellungen zeigen jedoch, dass sowohl die Simulationsgesamtheit, als auch die vereinfachte Approximation des Merkmals „Grundschülerinnen und Grundschüler" über das Alter der Populationseinheiten durchaus der Realität nahekommen. Der Grund dafür ist aber nicht zuletzt auch die Tatsache, dass die Basispopulation gezielt an den Zensusergebnissen von 2011 kalibriert wurde und es damit zu erwarten ist, dass grundlegende soziodemographische Merkmale und deren Verteilung mit einiger Sicherheit den realen Häufigkeiten dieses Jahres entsprechen.

\subsection{Geographische Referenzierung}

Die Zuordnung der Individuen des auf die eben beschriebene Art und Weise erstellten Basisdatensatzes an die real existierenden Adressen und damit auch gegebenenfalls in bestimmte Haushaltskonstellation stellt eine weitere Herausforderung dar, da zur räumlichen Verortung generell keine Informationen im synthetisch erzeugten Datensatz vorhanden sind. Um dennoch mit einiger Sicherheit die Personen der Basispopulation auf die real existierenden Adressen und Wohngebäude verteilen zu können, bieten sich die sogenannten Zensusgitterzellen an. Seit der Änderung des Bundesstatistikgesetzes im August 2013 wurde es der amtlichen Statistik rechtlich möglich, die Erhebungsmerkmale nicht mehr bis maximal auf die Ebene der Blockseite ${ }^{5}$, sondern - unabhängig von den Verwaltungseinheiten - auf Zellen, mit einer minimalen Gitterweite von 100Metern auszuwerten (Neutze 2015). Damit stehen Randverteilungen bestimmter Merkmale für diese $100 \mathrm{~m} \times 100 \mathrm{~m}$, beziehungsweise auf einer höheren Ebene $1000 \mathrm{~m} \times 1000 \mathrm{~m}$ großen Gitterzellen zur Verfügung. Somit besteht auch die Möglichkeit, die Individuen der Simulationsgesamtheit für Trier so auf die entsprechenden Gitterzellen zu verteilen, dass die für

\footnotetext{
5 Blockseite ist innerhalb eines Gemeindegebiets die Seite mit gleicher Straßenbezeichnung von der durch Straßeneinmündungen oder vergleichbare Begrenzungen umschlossenen Fläche. ( 10 Abs. 3 BStatG).
} 


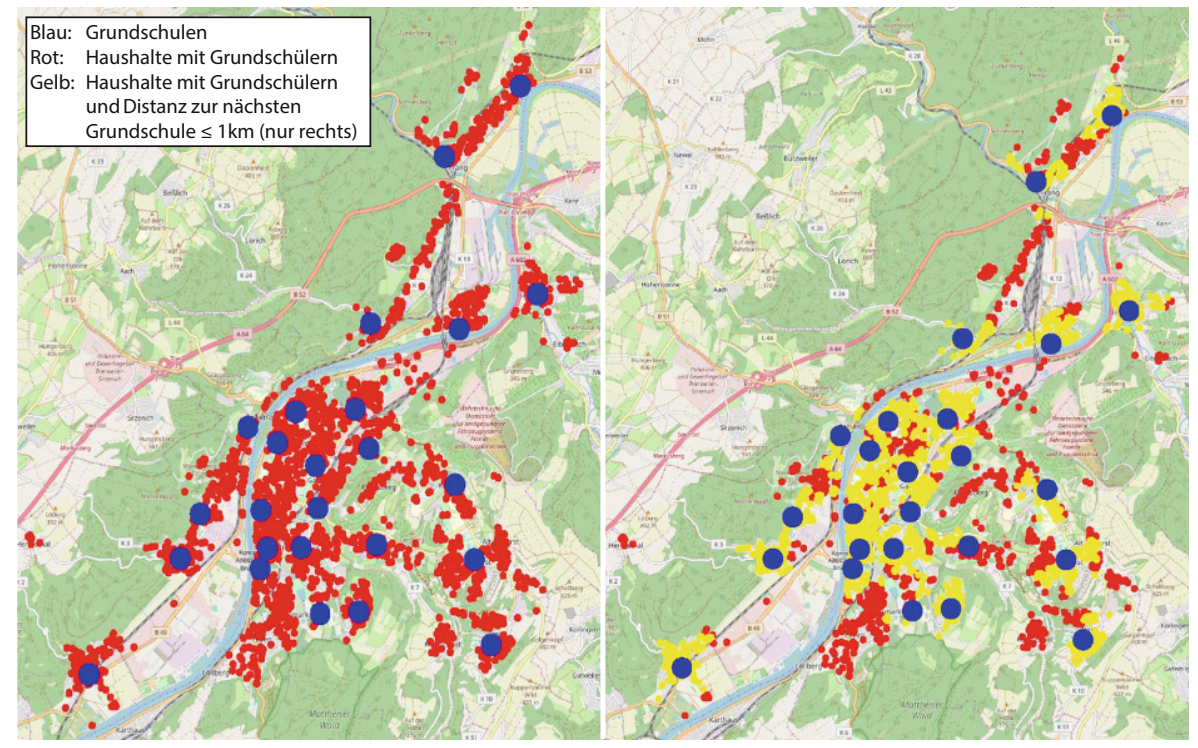

Abb. 2 Grundschulen und -schüler im Stadtgebiet (2011) (Map data copyrighted OpenStreetMap contributors and available from https://www.openstreetmap.org)

diese bekannten Randbedingungen bestmöglich nachgebildet werden. Dabei wurde in drei Stufen vorgegangen: Zunächst wurden die Personen auf die gröberen $1000 \mathrm{~m} \times 1000 \mathrm{~m}$ Zellen aufgeteilt, welche Informationen etwa zu Alter, Geschlecht und Wohnfläche der Personen enthalten. In einem zweiten Schritt wurden die Individuen von den gröberen Einheiten den deutlich kleineren $100 \mathrm{~m} \times 100 \mathrm{~m}$ Zellen zugeordnet. Als letztes wurden die Einheiten der Simulationsgesamtheit mit Hilfe von Kartenmaterial der OpenStreetMap Foundation innerhalb dieser Zellen auf real existierende Wohngebäude mit den entsprechenden Adressen verteilt (OpenStreetMap contributors 2021). Die Verteilung auf der letzten Stufe erfolgt auf Basis des verfügbaren Wohnraums, welcher von unbewohnten Gebäuden differenziert wird, zufällig (Burgard et al. 2019). Die kleinräumige Perspektive dieses Mikrosimulationsmodells erlaubt neben der Analyse der Entwicklung der absoluten Zahlen der Grundschülerinnen und Grundschüler auch Aussagen darüber, wo diese leben beziehungsweise leben werden. Dabei besteht das Ziel einer optimalen Versorgung eines Gebiets in Bezug auf die Grundschulen darin, eine möglichst bedarfsgerechte aber auch wohnortnahe Infrastruktur zu errichten. Zur Darstellung des Ergebnisses der Verortung der Population mit Hilfe der Gitterzellen wurden auf einer Karte alle Haushalte mit Kindern im Alter von sechs bis unter zehn Jahren und zusätzlich alle Grundschulen identifiziert und in Abb. 2 dargestellt. Generell zeigt sich hier, dass in Trier ein enges Netz an Schulen der Primarstufe vorhanden ist. Allerdings lassen sich auch Regionen erkennen, in denen Anfahrtswege und -zeiten länger sind.

Eben diese Betrachtung konkreter Anfahrtswege und -zeiten stellt eine spannende aber auch problembehaftete Erweiterung der hier vorgestellten kleinräumigen Perspektive dar. Um diese berechnen zu können, benötigt man eine Zuordnung der Grundschülerinnen und Grundschüler zu ihrer jeweiligen Schule. Hierbei zeigt sich 


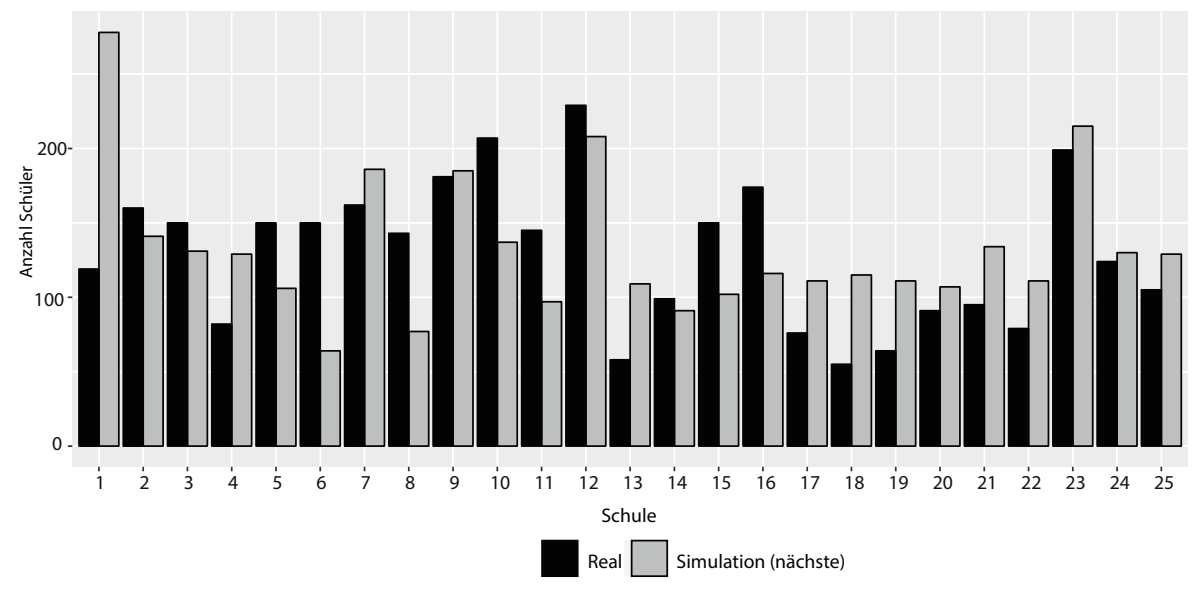

Abb. 3 Vergleich tatsächliche Zahl an Grundschülerinnen und Grundschülern je Einrichtung versus Zuordnung zu nächstgelegener. Die Einrichtungen wurden aus Gründen der Anonymität hier nur durchnummeriert

aber schnell ein entscheidendes Problem: Die Annahme, dass Grundschülerinnen und Grundschüler immer die nächstgelegene Schule besuchen führt - zumindest in diesem Beispiel in Trier - je nach Einrichtung zu einer deutlichen Über- oder Unterschätzung der Grundschülerzahl. Abb. 3 zeigt hier einen Vergleich der tatsächlichen Anzahl an Grundschülerinnen und Grundschülern auf Basis von Daten des kommunalen Bildungsmanagements von Trier und der Zahl an Schülerinnen und Schülern je Schule nach der Zuordnung der Simulationseinheiten zur jeweils nächstgelegenen Einrichtung. Grundlage waren hierfür die berechneten Anfahrtswege und -zeiten eines jeden Haushaltes mit einem Kind im Grundschulalter zu jeder Schule.

Die Gründe für die Über- oder Unterschätzung der Grundschülerinnen und Grundschüler in manchen Einrichtungen können dabei vielfältig sein: Zum einen gibt es administrative Grenzen in Form von Schulbezirken, die dafür sorgen, dass Schülerinnen und Schüler offenbar nicht immer die nächstgelegene Schule besuchen. Umgekehrt kann in Einzelfällen aber auch beantragt werden, dass ein Kind eben nicht die nächstgelegene Schule im Schulbezirk besucht, sondern beispielsweise eine, die näher am Arbeitsplatz der Eltern gelegen ist. Ebenfalls befinden sich im Stadtgebiet Trier zwei private Grundschulen, die unabhängig von administrativen Grenzen oder räumlichen Gegebenheiten Schülerinnen und Schüler anziehen und aufnehmen.

Man kann diese Informationen trotz der genannten Unschärfen natürlich dennoch nutzen, um auch anhand der Anfahrtswege und -zeiten Aussagen über die Versorgung der Stadt Trier mit Grundschulinfrastruktur zu treffen. Aufgrund der Problematik der Zuordnung muss man aber berücksichtigen, dass es sich dabei im Grunde nicht um eine Deskription der tatsächlichen Begebenheiten in Trier handeln kann, sondern viel mehr das Ergebnis einer Optimierung der Distanz der Schülerinnen und Schüler zu den Schulen dargestellt wird. Unter der Annahme, dass alle Grundschülerinnen und Grundschüler die ihnen nächstgelegene Einrichtung besuchen können ergibt sich ein durchschnittlicher Schulweg von 960Metern (Medi- 
an: 829 Meter) und eine Fahrzeit von 3,13 Minuten (Median: 2,93 Minuten). Erfolgt die Zuordnung hingegen unter Berücksichtigung der tatsächlichen Kapazitäten der Grundschulen, aber dennoch mit dem Ziel einer Minimierung der durchschnittlichen Anfahrtswege, so steigen diese Zahlen auf 1502 Meter (Median: 1006 Meter) beziehungsweise 4,07 Minuten (Median: 3,37 Minuten). Berücksichtigt man neben der Kapazität der Schulen dann auch noch die tatsächliche Anzahl an Schülerinnen und Schülern je Klassenstufe erhöhen sich die Zahlen wiederum auf 1644Meter (Median: 1033 Meter) beziehungsweise 4,34 Minuten (Median: 3,46 Minuten).

Auch wenn die starken Abweichungen zwischen arithmetischem Mittel und Median auf einige Ausreißer hindeuten, so zeigen diese Zahlen doch - unter der Annahme einer die Anfahrtswege minimierenden Zuordnung mit den genannten Einschränkungen hinsichtlich der empirisch festgestellten Kapazitäten in den Einrichtungen -, dass die Versorgung des Stadtgebietes mit Grundschulen angesichts der kleinen Anfahrtswege gut ist. Eine solche Betrachtung im Zeitverlauf ist aber aus zwei Gründen schwierig: Zum einen gehen diese Berechnungen mit einem erheblichen Rechenaufwand einher, sowohl was die Abfrage der Wege und Wegzeiten betrifft, als auch hinsichtlich der (bestmöglichen) Zuordnung der Schülerinnen und Schüler zu den Einrichtungen. Zum anderen werden generell mehr Daten benötigt um eben nicht nur ein Optimum beschreiben zu können, sondern auch tatsächliche Begebenheiten besser abbilden zu können. Solche Berechnungen sind also prinzipiell zukünftig möglich, konnten aber in dieser Forschungsarbeit nicht berücksichtigt werden.

\subsection{Mikrosimulation}

In Mikrosimulationsmodellen wird - in Abgrenzung zur klassischen Bevölkerungsvorausberechnung oder auch agenten-basierten Simulationsmodellen - versucht, Makrophänomene aufgrund des aus empirischen Daten abgeleiteten Verhaltens der Individuen auf der Mikroebene zu erklären. Da die vorliegende Basispopulation auf diese Weise in die Zukunft fortgeschrieben wird, handelt es sich um ein dynamisches Mikrosimulationsmodell. Änderungen der Einheiten der Basispopulation werden in zeitdiskreten oder periodenorientierten Fortschreibungen immer zu einem festen Zeitpunkt vorgenommen (Li und O'Donoghue 2013; Hannappel und Troitzsch 2015). Hierfür wird an jedem dieser Zeitpunkte geschätzt, mit welcher Wahrscheinlichkeit sich der Zustand eines Untersuchungsobjektes verändert. Der weitere Zeithorizont interessiert auch in dieser Analyse der Grundschulversorgung von Trier, da vor allem die Begebenheiten in der Zukunft als Entscheidungshilfe für politische Interventionen dienen können. Die Entscheidungen, die Grundschulversorgung in Trier mit Hilfe eines zeitdiskreten dynamischen Mikrosimulationsmodells zu analysieren, wurde dabei vor allem wegen der Möglichkeit getroffen, Wechselwirkungen zwischen verschiedenen Phänomenen und Mechanismen und deren Einfluss auf die Makrostrukturen beobachten zu können. Neben dieser grundlegenden Charakterisierung von Mikrosimulationen ist es notwendig, auch die konkrete Art und Weise der Fortschreibung näher zu betrachten. Wie bereits erwähnt werden bei einer periodenorientierten Modellierung die Eintritts- beziehungsweise Übergangswahrscheinlichkeiten aller Merkmale für jedes Individuum geschätzt und dann entschieden, ob sich ein Zustand verändert oder nicht. Der Ablauf eines jeden Simulationsschrit- 
tes ist dabei in Modulen organisiert. Eine solche Modulstruktur erlaubt es zudem, mit relativ geringem Aufwand und je nach Fragestellung verschiedene Variablen in neuen Modulen in die Simulation mit aufzunehmen, wodurch die erstellte Basispopulation für die Analyse einer Vielzahl von Problemstellungen nutzbar bleibt. Die Simulationsmodule repräsentieren biographische Ereignisse, wie beispielsweise Geburt, Heirat oder Tod, und strukturieren über Simulationsalgorithmen, ob ein Individuum in einem Modul berücksichtigt wird und wie der Fortschreibungsprozess konkret erfolgt. In ihnen sind somit auch die Fortschreibungsparameter enthalten, die wiederum aus dem Basisdatensatz selbst oder aus externen Untersuchungen berechnet wurden. Neben den Übergangswahrscheinlichkeiten ist ein weiterer zentraler Aspekt eines Simulationsmoduls die Durchführung von Zufallsexperimenten, was letztlich entscheidend dafür ist, ob eine Zustandsänderung bei einer Einheit stattfindet oder nicht. Die meisten Mikrosimulationsmodelle nutzen hierbei als probabilistische Fortschreibungstechnik die Monte-Carlo-Simulation wobei zufällig aus der Verteilung der Ereignisse gezogen wird (Galler 1997; van Imhoff und Post 1998). Hierbei werden die zugewiesenen Übergangswahrscheinlichkeiten für jedes Individuum kumuliert. Anschließend wird eine gleichverteilte Zufallszahl aus dem Intervall $[0,1]$ gezogen. Entsprechend der kumulierten Wahrscheinlichkeiten wird überprüft, in welchem Intervall die Zufallszahl liegt, um den Zustand der folgenden Periode zu bestimmen. Die Logik dieser probabilistischen Fortschreibung basiert dabei auf dem Gesetz der großen Zahlen, da bei wiederholter Durchführung der Simulation die realisierten Häufigkeiten gegen einen empirisch für dieses Ereignis ermittelten Erwartungswert konvergieren. Auch wenn sich deshalb annehmen lässt, dass bei gleichen Modulen und Fortschreibungsparametern auch die Ergebnisse mehrerer Simulationsdurchläufe auf der Makroebene annähernd gleich bleiben, handelt es sich dennoch um Ergebnisse stochastischer Natur, da auf der Mikroebene die Zufallszahlen immer wieder neu vergeben werden (van Imhoff und Post 1998; Hannappel und Troitzsch 2015). Das lässt sich an einem Beispiel verdeutlichen. So sei die empirisch ermittelte Wahrscheinlichkeit für eine Person innerhalb eines bestimmten Jahres zu sterben bei 10 Prozent. Bei einfacher Durchführung der Simulation kann die Person entweder sterben oder überleben. Wird die Simulation nun dreimal hintereinander durchgeführt, so besteht die Möglichkeit, dass die Person in allen Fällen überlebt (0 Prozent der Durchläufe), in einem Fall verstirbt (33,3 Prozent der Durchläufe), in zwei Fällen verstirbt (66,6\% der Durchläufe) oder in allen Fällen verstirbt (100 Prozent der Durchläufe). Der empirische Wert kann somit bei solch einer geringen Anzahl an Simulationsdurchläufen nicht erreicht werden. Bei einer höheren Anzahl an Wiederholungen konvergiert der Erwartungswert gegen die zugrundeliegende Wahrscheinlichkeit. Um die, aus den stochastischen Prozessen resultierende, Unsicherheit berücksichtigen zu können, sollten Mikrosimulationen grundsätzlich mit einer hohen Anzahl an Wiederholungen durchgeführt und die Ergebnisse anschließend gemittelt werden. Hier sind jedoch die Rechenzeit sowie die verfügbaren Kapazitäten zu beachten.

Einige der Module in einem Mikrosimulationsmodell sind zwingend notwendig, um eine annähernd realistische Fortschreibung der Basispopulation zu gewährleisten. Hierzu zählen beispielsweise Simulationsmodule, die die Mortalität in der Population strukturieren, ebenso wie jene, die neue Geburten prognostizieren. Andere 
Tab. 1 Simulationsmodule (in der entsprechenden Reihenfolge)

\begin{tabular}{ll}
\hline Modul & Beschreibung \\
\hline Geburten & Werden für Frauen im Alter zwischen 15 und 49 Jahren in zwei Stufen simuliert. In \\
& $\begin{array}{l}\text { einem ersten Schritt wird ermittelt ob es zu einer Geburt kommt, in einem zweiten } \\
\text { dann, ob es sich dabei um Zwillinge handelt. }\end{array}$
\end{tabular}

Mortalität

Hier wird die Sterbewahrscheinlichkeit auf Basis des Mikrozensus 2013/2014 unter Berücksichtigung soziodemographischer Merkmale geschätzt und dann je nach Alter und Geschlecht an die bekannten Werte der Periodensterbetafel angepasst.

Auszüge Kann einen Auszug aus dem elterlichen Haushalt beziehungsweise einer Wohngemeinschaft, ebenso wie durch eine Trennung umfassen. Im ersten Fall werden Auszugswahrscheinlichkeiten für Volljährige ermittelt und diese dann zunächst neuen Ein-Personen-Haushalten zugeordnet. Trennungen werden über spezifische Trennungswahrscheinlichkeiten geschätzt. Dann wird entschieden, welcher Partner den Haushalt verlässt. Sind Kinder in einem solchen Haushalt vorhanden wird zudem modelliert, ob sie bei einem der Partner verbleiben oder aufgeteilt werden und um welchen Partner es sich dann handelt.

Wanderungen Auch $\mathrm{Zu}$ - beziehungsweise Fortzüge werden anhand dieses Moduls und auf Basis differenzierter Wanderungstabellen für Rheinland-Pfalz in Abhängigkeit von verschiedenen Merkmalskombinationen wie Alter, Geschlecht und Staatsbürgerschaft geschätzt.

Entstehung neu- Die Entstehung neuer Haushalte erfolgt entweder durch die Gründung von Wohngeer Haushalte meinschaften oder über die Entstehung neuer Partnerschaften. Um die Partnerschaften zu simulieren werden dabei über ein Partner-Matching - welches auf verschiedene Arten erfolgen kann (Zinn 2012) - Personen aus der Basispopulation ausgewählt, die im Anschluss eine Beziehung eingehen.

Beziehungsstatus Hier lassen sich auch über Haushalte hinweg noch eindeutig Ehepartner über eine Identifikationsnummer bestimmen. Verwitwungen folgen dann deterministisch aus dem Tod des Ehepartners. Eine Heirat erfolgt nur wenn die Partner in einem Haushalt leben und ledig, geschieden beziehungsweise verwitwet sind. Geschieden werden können wiederum nur Paare, die vorher verheiratet sind.

Bildung und Er- Die Wahrscheinlichkeiten für die Erlangung eines Bildungsabschlusses oder den werbstätigkeit Übergang in die Erwerbstätigkeit resultieren maßgeblich aus dem Vorzeitpunkt. Hierbei wird darauf geachtet, dass keine unplausiblen Übergänge möglich sind.

Module wiederum dienen nur der Beantwortung von spezifischen Fragestellungen. Da es sich - wie bereits erwähnt - beim Übergang eines Kindes in die Grundschule nicht um eine komplexe Entscheidung, sondern um einen gewissermaßen durch das Alter determinierten Prozess handelt, wird auch zur Beantwortung dieser Frage zunächst kein spezifisches Modul benötigt, welches etwa eine Entscheidung für oder gegen einen Eintritt in die Primarstufe regeln würde. Für die Analyse der Grundschulversorgung werden daher insgesamt sieben Module herangezogen, die in erster Linie die demographische Entwicklung in Trier umfassend abbilden sollen. Diese sind mit einer kurzen Beschreibung in Tab. 1 dargestellt. ${ }^{6}$ Durch die Implementierung der Module zu Partnerschaften und Fertilität, welche durch spezifische Alignment-Methoden an die bekannten Entwicklungen angepasst wurden, kann angenommen werden, dass die Zahl der Kinder in Trier plausibel geschätzt und fortgeschrieben wird. Unter Alignment versteht man Methoden, die es erlau-

\footnotetext{
6 Die Module basieren dabei maßgeblich auf der Infrastruktur des MikroSim Simulationsmodelles (siehe Münnich et al. 2020) und in diesem Rahmen wurden auch die Module und Modelle bereits detaillierter diskutiert (siehe Münnich et al. 2021).
} 
ben entweder die Übergangswahrscheinlichkeiten direkt oder die zugrundeliegenden Parameter so zu kalibrieren, dass bekannte Totalwerte eingehalten werden (Burgard et al. 2020b).

Auch die Prognose des Bildungs- und Erwerbsstatus, sowie die Modellierung der Kohabitation sind durchaus relevant, da Partnerschaft und Fertilität eng mit diesen Dimensionen zusammenhängen (Hill und Kopp 2013). Hierbei liegen jedem Modul wiederum komplexe Modelle zur Schätzung der in den Simulationsalgorithmen integrierten Übergangswahrscheinlichkeiten zu Grunde.

Allerdings gibt es nicht nur einen Zusammenhang zwischen der Qualität der Module sowie Modelle selbst und dem letztendlichen Simulationsoutput, sondern es können sich auch Änderungen ergeben je nach Reihenfolge in der die Simulationsalgorithmen implementiert werden. Spätere Module nehmen durchaus die Veränderungen der vorangegangenen auf und die Übergangswahrscheinlichkeiten werden dadurch in Abhängigkeit zu den zuvor eingetretenen Ereignissen modifiziert. Daher muss also auch die Reihenfolge bei zeitdiskreten Simulationen berücksichtigt werden (Li und O'Donoghue 2013). Da in der durchgeführten Mikrosimulation hauptsächlich eine statische Modellierung für die Übergangswahrscheinlichkeiten verwendet wird, ist es hier aber weniger die Berücksichtigung der Reihenfolge selbst, sondern eher die Beachtung dieser im Modellierungsprozess der Bestimmung der Fortschreibungsparameter, welche grundlegend für den Umgang mit solchen abhängigen Ereignissen ist. Die Wahrscheinlichkeit zu heiraten ist zum Beispiel abhängig davon, ob man ein Kind bekommen hat und umgekehrt. Wird also das Modul der Geburten als erstes durchgeführt, muss der Beziehungsstatus der Person zum Vorjahreszeitpunkt mitberücksichtigt werden. Kommt es umgekehrt im ersten Modul zu einer Geburt, muss diese auch im Modul Beziehungsstatus berücksichtigt werden und einen Einfluss auf die Heiratsentscheidung haben können. Dennoch sollte versucht werden, eine inhaltlich möglichst sinnvolle Modulreihenfolge zu wählen, damit solche konkurrierenden Ereignisse am besten überhaupt nicht auftreten. Die Auflistung in Tab. 1 entspricht dabei auch der Simulationsreihenfolge, die die Basis für die Mikrosimulation der Grundschulversorgung in Trier darstellt.

\section{Dynamische Simulationsergebnisse}

\subsection{Benchmarking bis 2018}

Der eigentliche Sinn von Mikrosimulationsmodellen liegt, wie beschrieben, in der Fortschreibung einer Basispopulation in die Zukunft. Der Mehrwert entsteht nicht nur aus einer präzisen Gegenwartsanalyse, sondern auch aus einer validen Prognose der zukünftigen Begebenheiten. Hierzu ist natürlich zunächst eine Basispopulation notwendig, die den hohen Anforderungen von Mikrosimulationsmodellen gerecht wird. Das vorangegangene Kapitel hat dabei gezeigt, dass die Datenbasis grundsätzlich dazu geeignet ist in die Zukunft fortgeschrieben zu werden. Ebenfalls von großer Bedeutung für eine zuverlässige Prognose der Zukunft sind allerdings die bereits erwähnten Module mit ihren spezifischen Fortschreibungsparametern. Liegen diesen Fortschreibungsalgorithmen Modelle zugrunde, die nicht zu den realen Bedingun- 


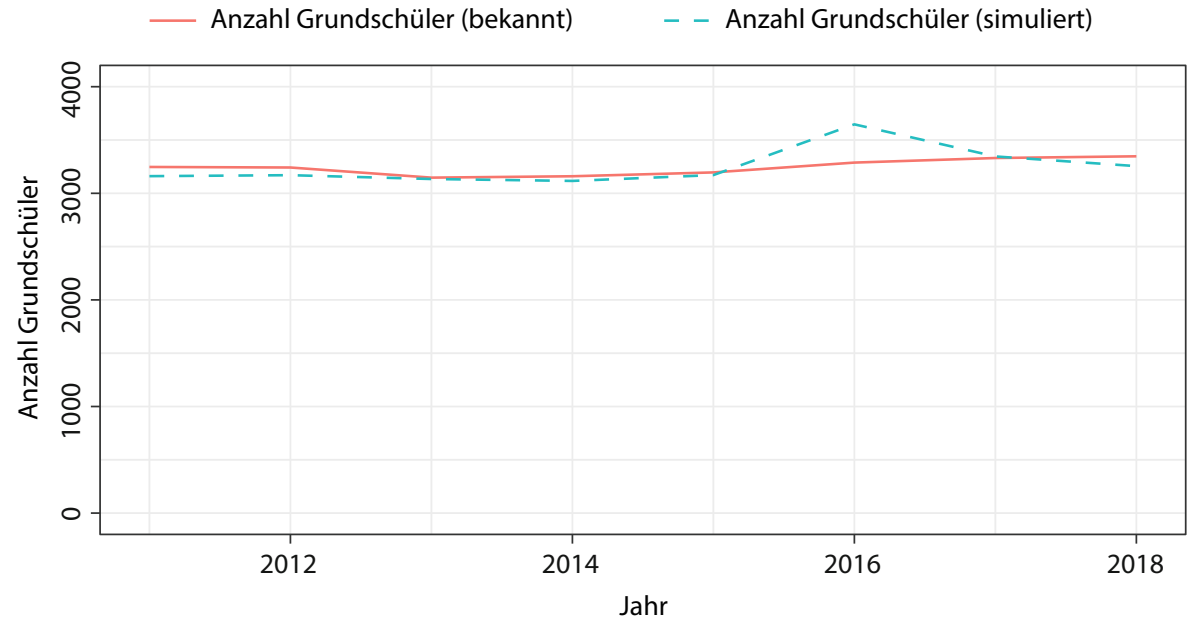

Abb. 4 Entwicklung der absoluten Gesamtgrundschülerzahl (2011-2018)

gen passen, zeigt sich in der Fortschreibung schnell, dass sich auch die Zahlen auf der Aggregatebene immer weiter von der Realität entfernen. Beobachtbar ist diese Diskrepanz aber natürlich nur dann, wenn auch realistische Daten überhaupt vorhanden sind, man die Fortschreibung also retrospektiv an Daten aus vergangenen Jahren evaluiert. Da die Basispopulation auf dem Zensus von 2011 basiert, stehen also prinzipiell Daten von diesem Zeitpunkt bis inklusive 2018 zur Verfügung, an denen man die Ergebnisse die eine Fortschreibung des Datensatzes produziert - und damit die Qualität der Module und Fortschreibungsparameter - testen kann. Mit Blick auf die leitende Fragestellung geht es dabei vor allem darum, die Anzahl an Schülerinnen und Schülern in Grundschulen und auch deren Verteilung auf die Klassenstufen zu prognostizieren. Schreibt man die Basispopulation in der beschriebenen Art und Weise fort, lohnt zunächst ein Blick darauf, ob die vorliegenden Zahlen zu den Gesamtgrundschülerzahlen in den Jahren 2011 bis 2018 auch annähernd mit der der Kinder im Alter von sechs bis neun Jahren in der Simulation übereinstimmen. Bei den absoluten Zahlenwerten der Simulationsergebnisse handelt es sich um die aufgerundeten Durchschnittswerte aus 30 Simulationsdurchläufen.

Abb. 4 zeigt, dass auch in der Fortschreibung die Zahl der simulierten Kinder im Alter von Sechs bis Neun anfangs unter der der tatsächlichen Grundschülerinnen und Grundschüler bleibt. Dies entspricht wiederum dem Bild, dass die Zahl der Kinder im Grundschulalter tatsächlich eher leicht niedriger ist, als die der in einer Grundschule eingeschulten. Wie bereits in Kap. 2 diskutiert, kann dies zum einen an der Problematik des tatsächlichen Einschulungsalters, an potentiellen Klassenwiederholungen oder an der Tatsache liegen, dass Pendler ihre Kinder auf einer Schule im Stadtgebiet anmelden. Während sich die Werte in den Jahren 2011 bis einschließlich 2015 allerdings noch in einer sehr ähnlichen Weise entwickeln, steigen die simulierten Schülerzahlen ab 2016 - bei einer fast gleichbleibenden tatsächlichen Zahl an Kindern im Grundschulalter - sprunghaft an. Dieser Diskrepanz verschwindet aber bereits im Folgejahr wieder gänzlich. Grund hierfür ist die Sondermigration 


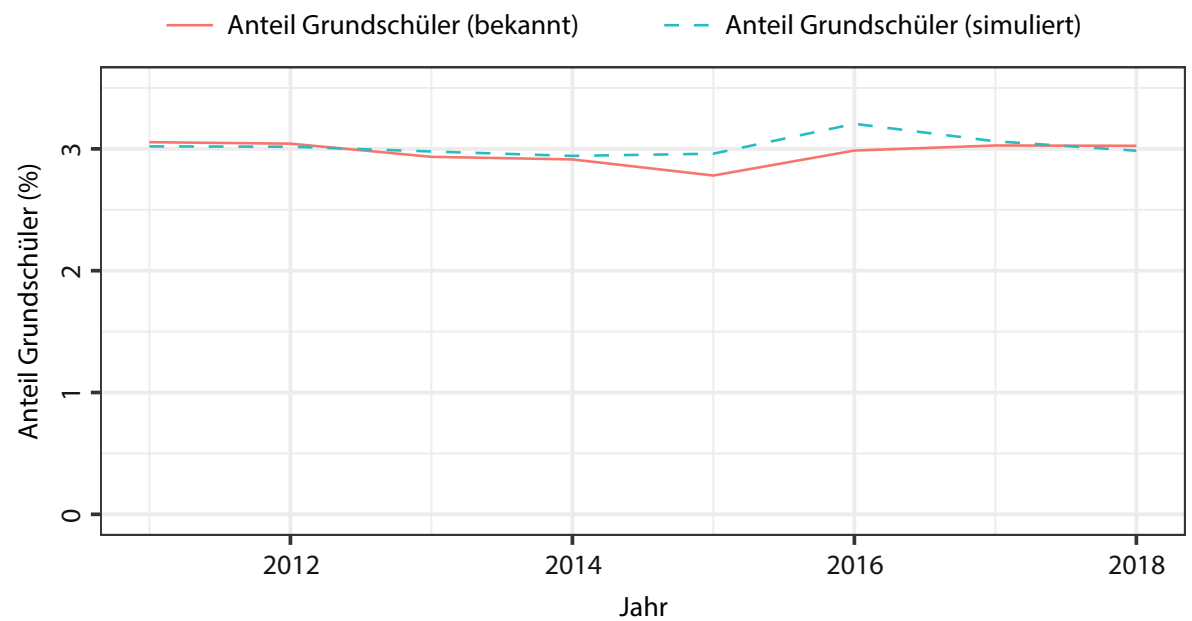

Abb. 5 Entwicklung der relativen Gesamtgrundschülerzahl (2011-2018) (Quelle: Kommunales Bildungsmanagement Trier und eigene Berechnungen)

im Rahmen der Flüchtlingsbewegung - 2016 wanderten deutlich mehr Menschen in die Erstaufnahmestelle in Trier zu, die aber 2017 bereits wieder im Rahmen der Weiterverteilung in die umliegenden Gemeinden abwanderten. Die Kinder im Alter zwischen sechs und neun Jahren, die wir als Grundschulkinder klassifizieren, welche in diesem Zeitraum in Trier lebten, wurden allerdings nicht in den Grundschulen, sondern in den Aufnahmeeinrichtungen beschult. Diese Sondermigration ist allerdings nicht nur bei den potentiellen Grundschülerinnen und Grundschülern, sondern auch in der gesamten Bevölkerung von Trier zu erkennen. Daher ist es sinnvoll, die absoluten Zahlen in das Verhältnis zur jeweiligen Bevölkerungszahl zu setzen. Auch in Abb. 5 kann man betrachten, dass die Kurven zu Beginn sehr ähnlich sind und später etwas auseinandergehen. Allerdings erstrecken sich die Differenzen auch hier nur auf die durch Sondermigration geprägten Jahre 2015 und 2016.

Der Anstieg des tatsächlichen Anteils der Grundschülerinnen und Grundschüler an der Gesamtbevölkerung weist dabei darauf hin, dass zwischen den Jahren 2015 und 2016 etwas stattgefunden hat, was dafür gesorgt hat, dass die Zahl der Schülerinnen und Schüler der Primarstufe überproportional zu der Gesamtbevölkerung gestiegen ist. Zum einen können hierfür Migrationsbewegungen in diesem Zeitraum verantwortlich sein. Zum anderen können aber auch wachsende Inklusionsbemühungen - die dafür sorgen, dass Kinder die vormals auf bestimmten Förderschulen eingeschult wurden nun häufiger eine Regelschule besuchen - einen solchen Anstieg mitverursachen. Sichtbar wird das beispielsweise daran, dass die Zahl der auf Förderschulen eingeschulten von 2007 bis 2017 um 21,8 Prozent abnahm (Ottenbacher 2018). Der Anstieg in Trier muss aber nicht zwangsweise auf die Geflüchteten aus den Kriegsgebieten im Nahen Osten zurückzuführen sein. Im Zuge der EU-Osterweiterung sind ab dem 01.01.2014 die Übergangsbestimmungen zur Freizügigkeit von Ländern wie Polen, Rumänien, Bulgarien und Ungarn entfallen (Ottenbacher 2018). Hierdurch hat sich ebenso wie durch den Zuzug von Geflüchteten die Zu- 
sammensetzung der ausländischen Bevölkerung in Trier durchaus in dieser Hinsicht verändert. Da es sich dabei um Zuwanderung aus dem EU-Ausland handelt, ist hier auch anzunehmen, dass diese ihren festen Wohnsitz in der Stadt Trier langfristig haben und somit auch ihre Kinder vermehrt die Grundschulen im Stadtgebiet besuchen. Dass die Simulationsergebnisse sich nicht komplett an die tatsächlichen Zahlen anpassen, liegt mit Sicherheit auch daran, dass es schwierig ist plötzlich auftretende externe Ereignisse - wie beispielsweise die Flüchtlingskrise - zu modellieren und in die Fortschreibung mit einfließen zu lassen. Wäre es in diesem Falle noch prinzipiell möglich die Basispopulation und die Fortschreibungsparameter an den vergangenen Ereignissen auszurichten und damit auch die Informationen für die Prognose in die Zukunft zu nutzen, ist es unmöglich Vorherzusagen, welche einschneidenden Schocks in Zukunft die reale Verteilung beeinflussen könnten. Jedoch bieten sich Mikrosimulationen an, um die Auswirkungen externer Schocks, die als Szenarien implementiert werden, sowohl unmittelbar als auch mittel- und langfristig zu untersuchen. Auch wenn die simulierten Gesamtschülerzahlen in Grundschulen leichte Abweichungen zeigen, lässt sich generell erkennen, dass bereits ohne die Berücksichtigung früherer beziehungsweise späterer Einschulungen oder der Abweichungen in der Dauer der Grundschulkarrieren, gute Ergebnisse mit dem Alter als Proxy-Variable für den Besuch einer Grundschule erreicht werden. Die Simulationsergebnisse sind also vergleichbar mit den tatsächlichen Begebenheiten. Hierbei sollte angemerkt werden, dass das primäre Ziel von Mikrosimulationen nicht in der Vorhersage von Punktprognosen liegt, da, neben der Komplexität des Gesamtmodells, externe Schocks wie beispielsweise Kriege und ihre Folgen, Pandemien oder Naturkatastrophen nicht antizipiert werden können. Die Fortschreibung einer Basispopulation mit den Methoden der Mikrosimulation erfolgt ab dem gegenwärtigen Zeitpunkt immer ceteris paribus. Der Sinn von Mikrosimulationsmodellen liegt daher hauptsächlich in der Möglichkeit der Untersuchung alternativer Szenarien (Li und O'Donoghue 2013).

\subsection{Zukünftige Entwicklung und Szenarien}

Relevanter als das nachbilden der bekannten Schülerzahlen ist natürlich die Fortschreibung in die Zukunft. Das Benchmarking hat dabei gezeigt, dass - unter Anpassung der Wanderungszahlen an bekannte Werte - die Grundschülerzahlen sehr gut abgebildet werden konnten, sodass mit einiger Überzeugung davon ausgegangen werden kann, dass die Fortschreibeparameter realistisch die Bevölkerungsentwicklung simulieren können. Zukünftig ist es aber nicht mehr möglich, den Wanderungssaldo in Trier an bekannten Werten auszurichten. Daher ist es Notwendig, Annahmen über die zukünftige $\mathrm{Zu}$ - beziehungsweise Abwanderung in Trier zu treffen.

In Hinblick auf die angesprochene Möglichkeit der Integration von Szenarien und das erwähnte Phänomen der Migration kann man nun die Entwicklung der Zahl der sechs bis neun Jahre alten Kinder in verschiedenen Wanderungsszenarien vergleichen. In Szenario 1 wurde angenommen, dass die Wanderungszahlen in Trier ab 2019 konstant auf den letzten bekannten Werten bleiben und die Nettowanderung damit insgesamt positiv ist (Zuwanderung: 13.532, Abwanderung: 12.510). In Szenario 2 wurde dann angenommen, dass ebenso viele Menschen zu- wie abwan- 


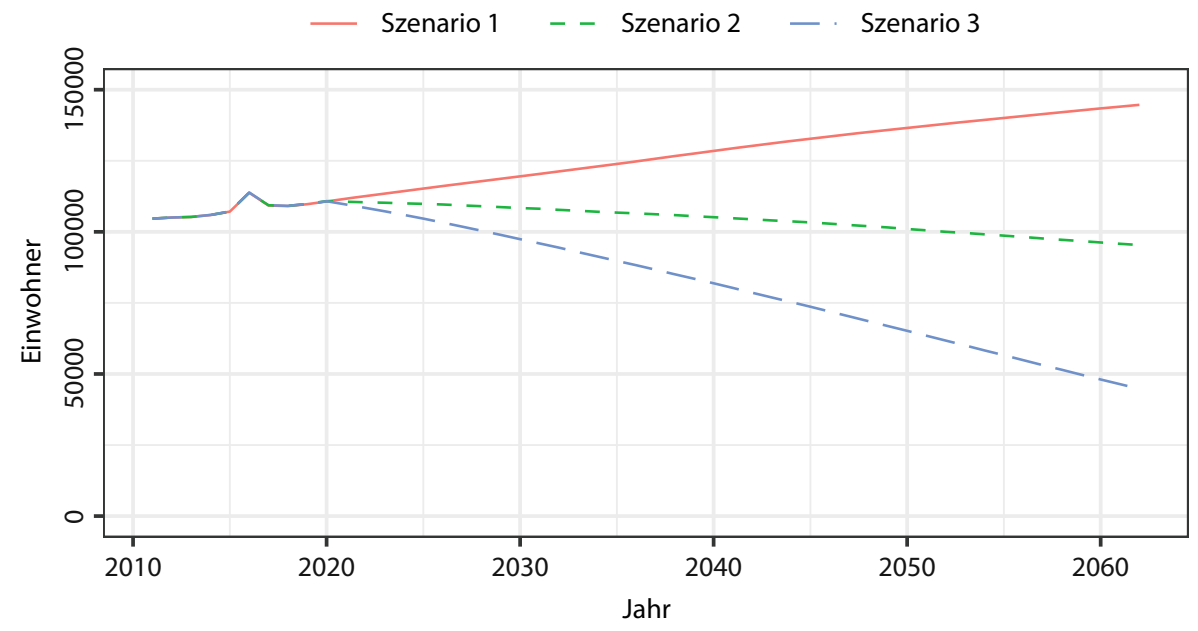

Abb. 6 Vergleich Wanderungsszenarien bis 2061 (Einwohnerzahl)

dern (Zuwanderung: 13.532, Abwanderung: 13.532), was eine Nettowanderung von 0 zur Folge hat. Abschließend wurden in Szenario 3 die letzten bekannten Wanderungszahlen einfach gedreht (Zuwanderung: 12.510, Abwanderung: 13.532), was eine insgesamt negative Nettowanderung bedeutet. Die bekannten Verteilungen (vor allem Alter und Geschlecht) unter den $\mathrm{Zu}$ - beziehungsweise Abwandernden wurden in allen drei Szenarien gleich auf dem Stand von 2019 belassen.

Auch wenn es sich bei diesen Zahlen grundsätzlich nur um beispielhafte Annahmen handelt, die einzig dazu dienen sollen zu illustrieren, wie solche Szenarien die zukünftige Entwicklung beeinflussen können, basieren die implementierten Szenarios also durchaus auf realistischen Wanderungszahlen, die auf empirisch beobachteten Werten beruhen.

Auch hier handelt es sich bei den Ergebnissen wieder um die Mittelwerte der Szenarien über 30 Simulationsdurchläufe pro Jahr. In Abb. 6 wird zunächst die Entwicklung der Gesamtbevölkerung dargestellt.

Die Möglichkeit Wanderung in den Jahren 2011 bis 2019 an bekannte Werte anzupassen führt dazu, dass die Kurven vor 2020 identisch verlaufen - die Szenarien wirken also erst ab dem Jahr 2020. Wenig überraschend zeigt sich danach, dass ein konstant positiver Wanderungssaldo in einem nahezu linearen Anstieg der Bevölkerungszahl Triers resultiert - und umgekehrt. Dass auch unter einer neutralen Nettowanderung Trier insgesamt schrumpft, liegt an Gründen wie einer Reproduktionszahl, die unter dem Bestandserhaltungsniveau liegt. Darüber hinaus weist Trier eine hohe Zuwanderung an jungen Menschen auf, die jedoch meist nur zu ihrer universitären Ausbildung in die Stadt ziehen und somit oft vor der Familiengründung wieder abwandern. Vor allem letzteres führt zu spezifischen Entwicklungen für Universitätsstädte, die sich auch auf die Zahl der Grundschülerinnen und Grundschüler (Abb. 7) auswirken. Es lässt sich auch beobachten, dass bei der Zuwanderung junger Erwachsener mehr Frauen in die Stadt ziehen. Das lässt sich mit der geisteswissenschaftlichen Ausrichtung der Universität erklären. Gleichzeitig ziehen Frauen mit 


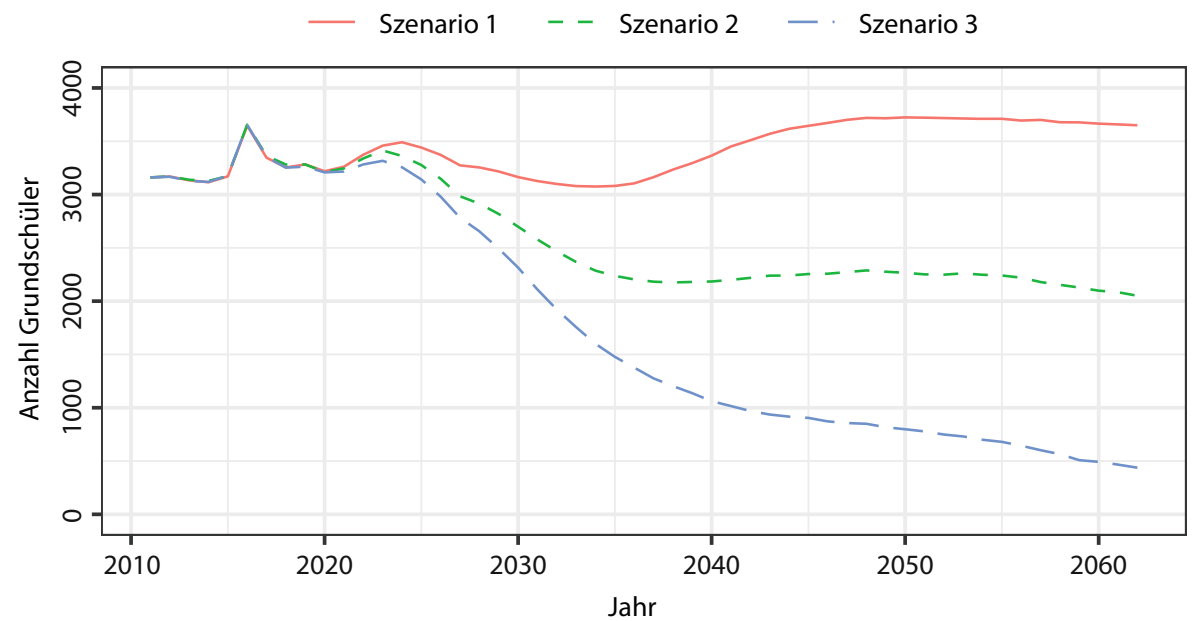

Abb. 7 Vergleich Wanderungsszenarien bis 2061 (Anzahl Grundschülerinnen und Grundschüler)

leicht fortgeschrittenem Alter überproportional häufig wieder fort, sodass letztendlich langfristig mehr zugewanderte Männer in Trier verbleiben.

Generell lassen sich ähnliche Tendenzen wie bei der Einwohnerzahl konstatieren - die höchste Grundschülerzahl resultiert aus dem Szenario mit der höchsten Nettowanderung. Allerdings lässt sich nun feststellen, dass die Kurven deutlich nichtlinear verlaufen. In Szenario 1 lässt sich zwar ein deutlicher Anstieg der Einwohnerzahl erkennen, während die Grundschülerzahl selbst an ihrem Maximum kaum über den bereits 2016 erreichten Werten liegt. Grund hierfür, wie auch für die wellenförmige Entwicklung unter Szenario 1 (und bedingt auch Szenario 2), kann ebenfalls die hohe Abwanderung von Menschen kurz vor der typischen Phase der Fertilitätsentscheidungen sein. Erst wenn eine gewisse Zahl an jungen Menschen nach Trier gezogen und dort sesshaft geworden ist, zeigt sich auch ein beinahe sprunghafter Anstieg der Grundschülerzahlen (ab ca. 2035). Dieser ist vermutlich auf die Kinder der jungen Menschen zurückzuführen, die jetzt eben zu einem geringeren Anteil abwandern.

Diese Kohorte der Neugeborenen kann man in Szenario 2 auch noch erahnen, wohingegen in Szenario 3 ein kontinuierlicher Rückgang der Grundschülerzahlen zu beobachten ist. Während der Rückgang bis 2040 sehr steil verläuft, wird die Kurve anschließend flacher.

Neben den für die konkrete Planung der Grundschulinfrastruktur ausschlaggebenden absoluten Grundschülerzahlen reflektiert der Anteil der Grundschülerinnen und Grundschüler an der Gesamtbevölkerung (Abb. 8) - und damit die Kombination der vorangegangenen Grafiken - eher die Entwicklung der Bevölkerungsstruktur im Ganzen.

Auffällig ist, dass der Grundschüleranteil in allen drei Szenarien insgesamt sinkt, was an bekannten demographischen Prozessen zu liegen scheint. Das Szenario 1 und Szenario 2 wiederum einen sehr ähnlichen Verlauf zeigen und insgesamt ein gewisser Niveauunterschied besteht, zeigt, dass nicht nur Menschen jenseits der fertilen Phase vermehrt nach Trier ziehen, sondern vor allem auch junge Menschen bezie- 


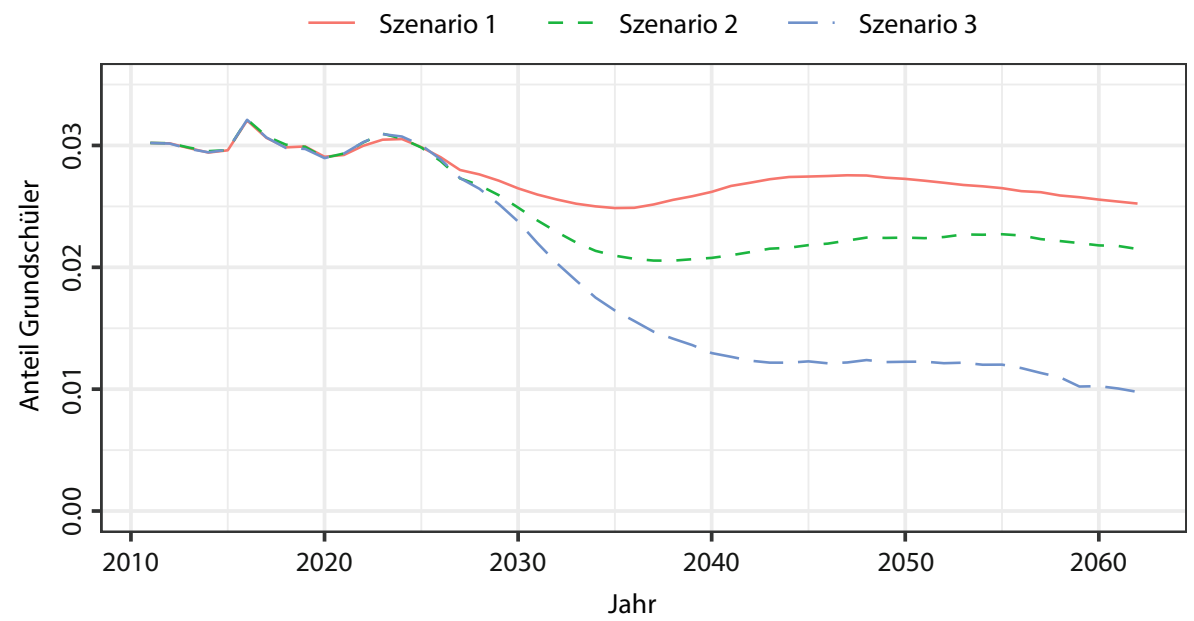

Abb. 8 Vergleich Wanderungsszenarien bis 2061 (Anteil Grundschülerinnen und Grundschüler)

hungsweise Familien. Umgekehrt wandert diese Bevölkerungsgruppe in Szenario 3 anscheinend überproportional ab. Sichtbar wird dies am konstanten abnehmen des Anteils der Grundschülerinnen und Grundschüler an der Gesamtbevölkerung auf unter 1 Prozent.

Letztlich können auf Grundlage des Mikrosimulationsmodelles neben der Migration, welche die Zahl der Grundschülerinnen und Grundschüler unmittelbar beeinflusst, auch weitere inhaltliche Szenarien einander gegenübergestellt werden. Diese Szenarien können dabei entweder auf fiktiven Annahmen über zukünftige Entwicklungen, auf empirisch erwartbaren Trends oder aber auch auf konkreten politischen Bemühungen zur Gestaltung der Zukunft beruhen. Auch Wanderungsszenarien können sehr präzise formuliert werden. So lassen sich Annahmen bezüglich aller soziodemographischen und sozioökonomischen Merkmale der zu- bzw. abwandernden Bevölkerung detailliert in die Simulation integrieren.

Das Potential der Methode liegt also nicht nur in der Analyse der Grundschulversorgung von Trier und der Prognose der Strukturen unter der Bedingung diverser inhaltlicher Szenarien, sondern ganz allgemein in der Möglichkeit bei diversen Themenschwerpunkten Entscheidungsträgern in der Praxis Informationen auf der Basis von fundierten Prognosen zu geben. Aber auch wissenschaftliche Theorien - vor allem Handlungstheorien - können mit Hilfe von Mikrosimulationsmodellen verglichen und auf ihre potentielle Bedeutung für die zukünftige Entwicklung der Gesellschaft hin analysiert werden.

\subsection{Entwicklung der räumlichen Verteilung der Grundschülerinnen und Grundschüler in Trier}

Mit Blick auf die Grundschulversorgung kann man mit einem kleinräumigen Simulationsmodell wie es hier vorliegt auch untersuchen, wo im Stadtgebiet zukünftig Schwerpunkte entstehen oder wo vorhandene Einrichtungen mit schwindenden 
Schülerzahlen zu kämpfen haben. Identifiziert man in einer Basispopulation ein Neubaugebiet, in dem sich innerhalb kürzester Zeit viele junge Paare ansiedeln, so ist zu erwarten, dass in den Folgejahren dort auch eine erhöhte Zahl an Kindern im Grundschulalter lebt. Speziell für diese dann aber dort eine Bildungseinrichtung zu planen, kann unter Umständen nicht ratsam sein, da wiederum nach einigen Jahren diese Kinder die Grundschule absolviert haben und auch nicht zwangsweise abzusehen ist, dass in diesem Gebiet in naher Zukunft wieder ein solcher Geburtenboom stattfindet. Auf diese Weise kann man dann nicht nur die erwarteten Folgen von alternativen Szenarien auf die aggregierte Zahl der Grundschülerinnen und Grundschüler analysieren, sondern auch eine räumliche Komponente betrachten.

Vor allem mit Blick auf die verschiedenen Stadtgebiete, die zwar nach innen oftmals in Bezug auf zentrale soziodemographische Merkmale sehr homogen sind, sich aber untereinander deutlich unterscheiden können, ist zu erwarten, dass die Entwicklungen in diesen durchaus voneinander abweichen. Dadurch kann sich auch die Verteilung der Kinder im Grundschulalter im Stadtgebiet deutlich verändern. Gebiete, in denen derzeit kaum Kinder zwischen sechs und neun Jahren leben und die generell einen sehr hohen Altersdurchschnitt aufweisen, könnten in Zukunft von jungen Familien neu besiedelt werden, wohingegen andere Stadtteile, in denen derzeit viele Kinder geboren wurden, zunächst keinen erneuten Anstieg der Geburtenzahlen erwarten dürften. Solche Mechanismen führen in einer langfristigen Betrachtung dazu, dass sich das Stadtbild durchaus mehrmals verändern kann. Allerdings gibt es auch für eine solche Betrachtung derzeit noch Beschränkungen, da das Phänomen der Binnenwanderung im Stadtgebiet noch nicht wirklich in den Modulen erfasst werden kann. Außerdem konnten Neubaugebiete, die explizit ausgewiesen werden müssen, zum jetzigen Stand noch nicht berücksichtigt werden. Es ist zwar durchaus sinnvoll anzunehmen, dass junge Familien nach der Geburt ihres Kindes ihre derzeitige Wohnung verlassen und in eine größere und günstigere Alternative in anderen Stadtteilen umziehen, allerdings ist dies im zugrundeliegenden kleinräumigen Mikrosimulationsmodell noch nicht umgesetzt.

Auf einer gröberen Ebene können geglättete Schätzungen der Wahrscheinlichkeitsdichte über bivariate Kerndichteschätzungen dazu herangezogen werden, um Muster und Veränderungen in der Struktur der Stadt aufzuzeigen und erste Anhaltspunkte für eine bedarfsgerechte Grundschulplanung zu liefern. Für weitere Informationen zur Verwendung von Kerndichteschätzungen zur Darstellung von Zusammenhangs- und Konzentrationsgebieten sei beispielsweise auf Rendtel und Ruhanen (2018) und Rendtel et al. (2021) verwiesen. Zudem kann man mit einer Kerndichteschätzung die Verteilung der Haushalte mit Kindern im Grundschulalter besser veranschaulichen. Damit lassen sich Ballungsgebiete innerhalb von Verwaltungsgrenzen identifizieren. Diese Betrachtungsweise, die es dann auch erlaubt einzelne Ausreißer nicht zwingend mit zu berücksichtigen, ist in Abb. 9 dargestellt und zwar einmal für das Startjahr 2011 und einmal für einen beispielhaften Durchlauf pro Szenario im Jahr 2061. Somit ist es auch möglich zu analysieren, ob und wie die Wanderungsszenarien Einfluss auf die räumliche Verteilung der Grundschülerinnen und Grundschüler nehmen und was dies für die verschiedenen Grundschulen (Dreiecke) bedeutet. 


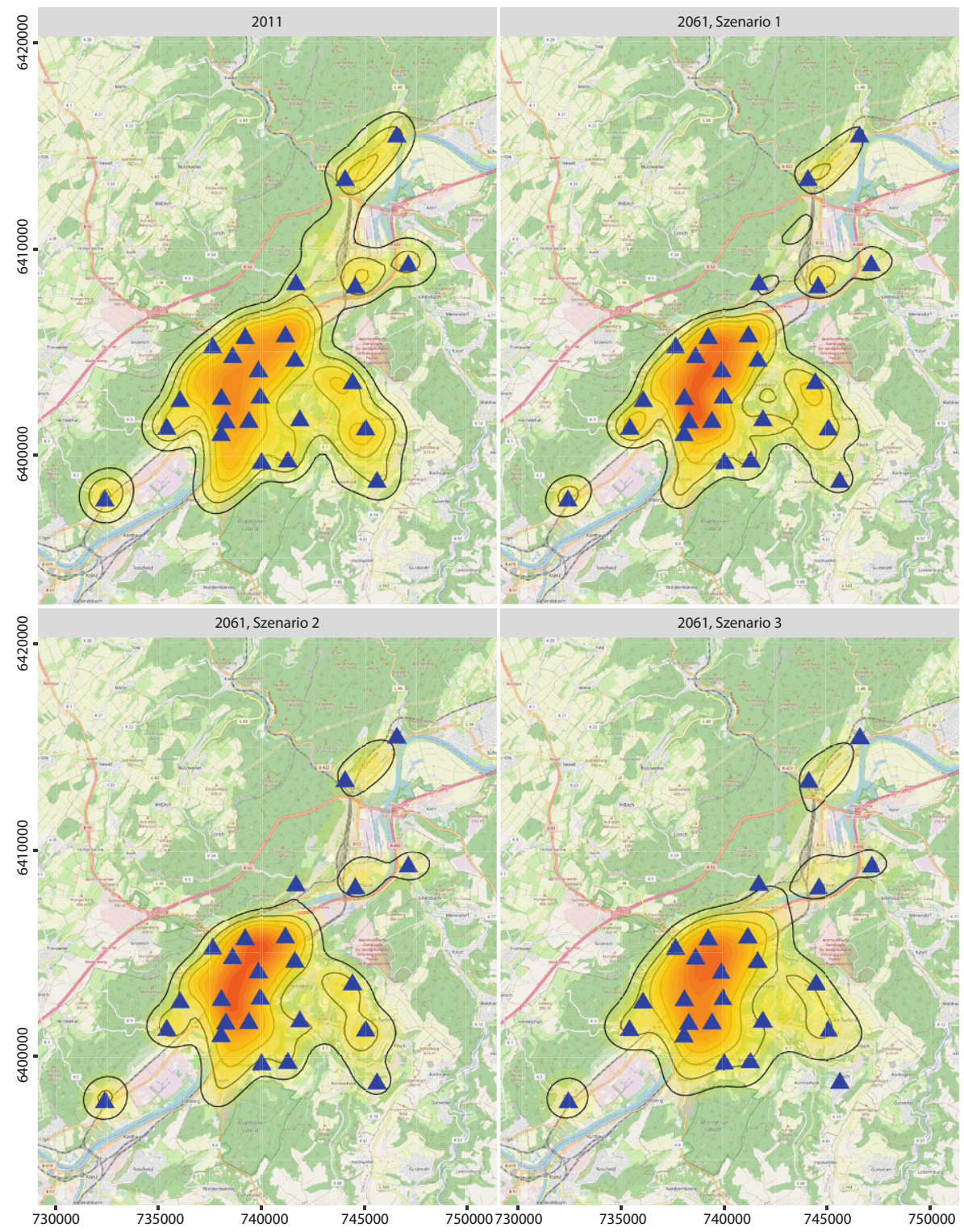

Abb. 9 Räumlicher Vergleich der Szenarien (Kerndichteschätzer) (Map data copyrighted OpenStreetMap contributors and available from https://www.openstreetmap.org)

Die Nutzung von Dichteschätzern bietet sich dabei aus mehreren Gründen an: Sie erlauben nicht nur eine effektive Darstellung des Zusammenhangs von Konzentrationsgebieten auch für bestimmte Bevölkerungsgruppen, sondern vermeiden in Abgrenzung zu den gängigen Flächenkartogrammen - Sprünge an den Gebietsgrenzen und sind flächentreu (Rendtel und Ruhanen 2018). Je dunkler die Färbung 
der Flächen in Abb. 9, desto höher ist die Konzentration an Grundschülerinnen und Grundschülern in einem Gebiet.

An diesen Grafiken (Abb. 9) lässt sich das Potential erkennen, welches eine kleinräumige Perspektive hat, da durchaus interpretierbare Veränderungen sichtbar werden. Ganz allgemein lässt sich zeigen, dass die Zahl der Grundschülerinnen und Grundschüler im Vergleich zum Basisjahr 2011 in allen Szenarien dazu tendiert sich in der Stadtmitte zu konzentrieren, was sich an der intensiveren Färbung des Kerndichteschätzers in diesem Bereich erkennen lässt. Die Konzentration der Grundschülerinnen und Grundschüler in den Randgebieten hingegen scheint ebenfalls in allen Szenarien eher abzunehmen, was konträr durch eine weniger intensive oder gar nicht mehr vorhandene Einfärbung erkennbar ist. Auch zwischen den Szenarien lassen sich vor allem in den weiter vom Stadtgebiet entfernten Stadtteilen Unterschiede erkennen. Nimmt man die Konzentration als Maßstab für die Auslastung einer Grundschule (Dreiecke), dann finden sich in Szenario 1 noch an allen Grundschulen Kinder im Grundschulalter, während im Szenario 2 und 3 Grundschulen durchaus an Standorten stehen, an denen kaum oder gar keine Schülerinnen und Schüler mehr leben. Allerdings stehen die Grundschülerinnen und Grundschüler bei Kerndichteschätzungen immer in Relation zur Gesamtbevölkerung, sodass diese Grafiken zwar allgemeine Informationen zu Konzentrationsgebieten geben können, weniger aber dazu geeignet sind, die Basis für die tatsächliche Grundschulplanung zu sein. Daher zeigen die folgenden Abbildungen (Abb. 10) die Anzahl der Grundschülerinnen und Grundschüler im Stadtgebiet. Ist ein Hexagon in der Grafik vorhanden, so gibt es an dieser Stelle mindestens ein Kind im Alter zwischen sechs und neun Jahren. Je mehr Kinder innerhalb eines Hexagons in diesem Alter sind, desto dunkler ist es dargestellt.

Diese Grafiken weisen im Gegensatz zu den Kerndichteschätzern darauf hin, dass in Szenario 2 und Szenario 3 die Zahl der Grundschülerinnen und Grundschüler flächendeckend abnimmt, auch wenn immer noch die meisten in der Stadtmitte zu finden sind. In Szenario 3 kann man sogar erkennen, dass in randständigen Gebieten (vor allem im Nord-Osten und im Süd-Westen der Stadt) viele Hexagone sogar gänzlich verschwinden - hier also gar keine Grundschülerinnen und Grundschüler mehr zu finden sind. Szenario 1 und damit das einzige Szenario, in welchem die Anzahl der Grundschülerinnen und Grundschüler nicht sinkt, sondern leicht steigt, zeigt hingegen, dass gerade im Nord-Osten ein Zuwachs zu verzeichnen ist. Insgesamt steigt die Zahl der Grundschülerinnen und Grundschüler aber auch hier am meisten im Innenstadtbereich an.

Mit Blick auf die Möglichkeit verschiedene Szenarien testen zu können, können sich diese Ergebnisse allerdings auch gravierend ändern. Integriert man ein Modul zur Binnenwanderung, welches diese als abhängig von den Mietpreisen berücksichtigt, so könnte ein durchaus realistisches Szenario lauten, dass die Kosten für Mietwohnungen in der Stadtmitte kontinuierlich ansteigen. Junge Familien wiederum brauchen oftmals große und bezahlbare Wohnungen, die in diesem Fall eher nicht im Zentrum der Stadt zu finden sind. Unter diesen Umständen nehmen wir umgekehrt an, dass die Konzentration an Grundschülerinnen und Grundschülern eher in den Randgebieten stattfindet und sich somit auch dort Grundschulen befinden müssten, sofern diese möglichst wohnortnah sein sollen. Neben spezifischen Szenarien 

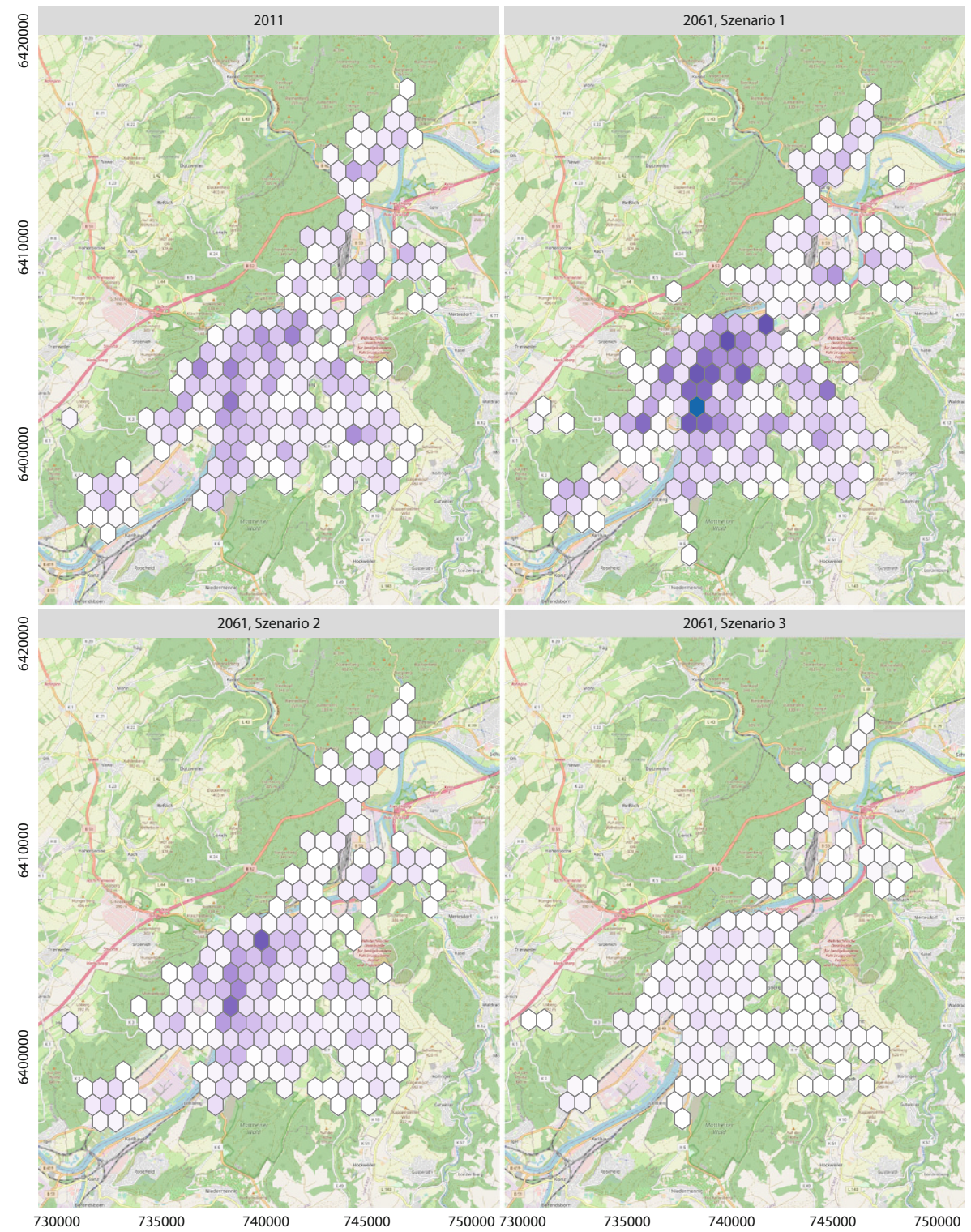

Abb. 10 Räumlicher Vergleich der Szenarien (Hexplots) (Map data copyrighted OpenStreetMap contributors and available from https://www.openstreetmap.org)

die Einfluss auf die grundsätzliche Zahl der Kinder im Grundschulalter haben, können so also zukünftig auch spezifische Fragestellungen bearbeitet werden, die sich dezidiert damit beschäftigen, in welchen Gebieten der Stadt sich Grundschulkinder befinden. 


\section{Fazit und Ausblick}

Im Zentrum dieser Arbeit standen vorrangig zwei Fragestellungen: Zum einen sollte eine Sachanalyse der Grundschulversorgung in Trier vorgenommen werden, zum anderen sollte die relativ neue Methodik der kleinräumigen Mikrosimulationsmodelle in einer konkreten Anwendung evaluiert werden.

Die erste Fragestellung der Arbeit lässt sich zunächst leicht beantworten: Die Grundschulversorgung in Trier ist gut und das sowohl in Hinblick auf die zur Verfügung gestellten Plätze, als auch auf die Verteilung der Einrichtungen im Stadtgebiet. Diese Schlussfolgerung ergibt sich maßgeblich aus der Analyse der Begebenheiten im Startjahr 2011 (siehe Kap. 2.2) und der Feststellung, dass sich weder die absolute Zahl, noch die Konzentration in einzelnen Bereichen der Stadt im Simulationsverlauf - über alle Szenarien hinweg - maßgeblich nach oben verändert. Ein solches Ansteigen der Grundschülerzahlen würde bedeuten, dass neue Kapazitäten notwendig wären um zumindest das Niveau der Startperiode zu erhalten, eine höhere Konzentration in einem bestimmten Stadtgebiet als im Jahr 2011 wiederum würde sich negativ auf Anfahrtswege und -zeiten auswirken. Da aber beides nicht der Fall ist kann davon ausgegangen werden, dass sich zumindest diese beiden Parameter eher sogar weiter verbessern. Dies ist auch nicht unbedingt verwunderlich, da es sich um ein dicht besiedeltes städtisches Gebiet handelt. Eine Gegenüberstellung der Stadt mit angrenzenden und eher ländlich geprägten Landkreisen sollte daher in zukünftige Betrachtungen und Planungen integriert werden und soll auch ein Teil der weiteren Arbeiten zu diesem Projekt sein. Auch ein kurzer Blick in die zukünftigen Entwicklungen zeigt, dass eher nicht damit zu rechnen ist, dass gravierende Herausforderungen auf das Bildungsmanagement der Stadt zukommen, da die Zahl an Grundschülerinnen und Grundschülern ab einem Höhepunkt im Jahr 2025 eher rückläufig ist. Selbst unter einem Wanderungsszenario mit konstant positiver Nettowanderung steigt die maximale Anzahl der Grundschülerinnen und Grundschüler nur leicht an. Kritisch ist jedoch anzumerken, dass diese Aussagen nur unter der Annahme einer gewissen Konstanz in der Grundschulinfrastruktur gültig sind. Ändert sich diese, beispielsweise durch die Schließung einer oder mehrerer Einrichtungen, so kann dies unter Umständen eine mitunter drastische Verschlechterung der allgemeinen Versorgung nach sich ziehen.

Auch die kleinräumige Betrachtung zeigt, dass sich nicht wider Erwarten in einem bestimmten Stadtgebiet ein deutlicher Mehrbedarf an Grundschulen ergibt. Generell wirken sich alle Szenarien durchaus flächendeckend aus. Die Konzentration ist und bleibt dennoch unter allen Szenarien im Innenstadtbereich am höchsten. Ein positiver Wanderungssaldo führt außerdem dazu, dass in Randgebieten die Zahl der Grundschülerinnen und Grundschüler steigt - allerdings gibt es auch hier schon entsprechende Einrichtungen um diesen Bedarf prinzipiell zu decken. Der flächendeckende Rückgang an Schülerinnen und Schülern mit einer Reduzierung der Zahl auf etwas mehr auf die Hälfte (Szenario 2) führt aber dennoch höchstwahrscheinlich dazu, das einzelne Schulen schließen müssen und dadurch deutlich längere Anfahrtswege und -zeiten entstehen.

Die Beantwortung der zweiten Frage über die Eignung der Methode der kleinräumigen Mikrosimulationsmodelle für eine derartige Fragestellung gestaltet sich 
demgegenüber etwas schwieriger. Generell wurde gezeigt, dass solche Analyseinstrumente interessante und wertvolle Erkenntnisse liefern können. Dies bezieht sich dabei auch nicht ausschließlich auf die Produktion von Fakten für politische Interventionen, sondern auch auf die Möglichkeit der Bearbeitung wissenschaftlicher Problemstellungen. Sowohl eine retrospektive Evaluation von bestimmten postulierten (theoretischen) Modellen, als auch die Abschätzung von nicht intendierten Nebenfolgen stellen hier zentrale Anwendungsfelder für die Forschung dar. (Manzo 2007; Saam 2015) Dennoch ist es, vor allem mit Blick auf das Vorhaben des übergeordneten MikroSim-Projektes ein bundesweites Mikrosimulationsmodell zu erstellen, notwendig, die einzelnen Bestandteile zu verbessern und auch mit Hilfe weiterer Datensätze und Informationen zu erweitern.

Dies gilt vor allem für die weniger direkt mit der Frage nach der Grundschulversorgung verbundene Wanderungsthematik. Die Veränderungen, die Wanderung auf die gesamte Bevölkerungs- und Sozialstruktur hat, wurden in den untersuchten Szenarien mehr als deutlich. Gerade in Trier spielen zwei Faktoren eine zentrale Rolle, Trier als Grenzgebiet zu Luxemburg, in dem die Haus- und Wohnungspreise erheblich höher sind, und als Studentenstadt (Universitätsstadt). Die jüngsten Entwicklungen im Zuge von Sondermigration müssen unbedingt in den Modellen berücksichtigt werden. Ebenso ist von nicht homogenen Fortschreibungsprozessen zwischen verschiedenen Gruppen auszugehen. Diese beziehen sich beispielsweise auf Fertilität und Partnerwahl.

Generell kann dabei auf eine breite Forschung im Bereich der Familiensoziologie zurückgegriffen werden (siehe Hill und Kopp (2013) für einen Überblick über den Forschungsstand). Zudem ist ein sensibler Umgang mit den kulturellen und gesellschaftlichen Differenzen und den daraus resultierenden Unterschieden im Fertilitätsverhalten der autochthonen und nicht-autochthonen Bevölkerungsgruppen notwendig. Ziel ist es dabei nicht nur eine realitätsnahe Fortschreibung der Basispopulation zu erreichen, sondern auch die Zahl der Grundschülerinnen und Grundschüler prognostizieren sowie möglicher weiterer infrastruktureller Aspekte beantworten zu können.

Danksagung Der Artikel basiert auf Forschungsarbeiten im Projekt REMIKIS sowie in der DFG Forschungsgruppe FOR 2559 MikroSim. Unser besonderer Dank gilt der Nikolaus-Koch-Stiftung sowie der Deutschen Forschungsgemeinschaft für die Förderung, welche die Forschungsarbeiten für diesen Artikel ermöglicht haben. Weiterer Dank gilt den Kolleginnen und Kollegen in beiden Forschungsprojekten, welche durch zahlreiche Diskussionen und konstruktive Kritiken zur Verbesserung des Artikels beigetragen haben.

Funding Open Access funding enabled and organized by Projekt DEAL.

Open Access Dieser Artikel wird unter der Creative Commons Namensnennung 4.0 International Lizenz veröffentlicht, welche die Nutzung, Vervielfältigung, Bearbeitung, Verbreitung und Wiedergabe in jeglichem Medium und Format erlaubt, sofern Sie den/die ursprünglichen Autor(en) und die Quelle ordnungsgemäß nennen, einen Link zur Creative Commons Lizenz beifügen und angeben, ob Änderungen vorgenommen wurden.

Die in diesem Artikel enthaltenen Bilder und sonstiges Drittmaterial unterliegen ebenfalls der genannten Creative Commons Lizenz, sofern sich aus der Abbildungslegende nichts anderes ergibt. Sofern das betreffende Material nicht unter der genannten Creative Commons Lizenz steht und die betreffende Handlung 
nicht nach gesetzlichen Vorschriften erlaubt ist, ist für die oben aufgeführten Weiterverwendungen des Materials die Einwilligung des jeweiligen Rechteinhabers einzuholen.

Weitere Details zur Lizenz entnehmen Sie bitte der Lizenzinformation auf http://creativecommons.org/ licenses/by/4.0/deed.de.

\section{Literatur}

Burgard JP, Krause J, Merkle H, Münnich R, Schmaus S (2019) Conducting a dynamic microsimulation for care research: data generation, transition probabilities and sensitivity analysis. In: Steland A, Rafajłowicz E, Okhrin O (Hrsg) Workshop on Stochastic Models, Statistics and their Application Dresden, Germany, 03.2019 Springer, Cham, S 269-290

Burgard JP, Dieckmann H, Krause J, Merkle H, Münnich R, Neufang KM, Schmaus S et al (2020a) A generic business process model for conducting microsimulation studies. Stat Transit New Ser 21(4):191-211

Burgard JP, Krause J, Schmaus S (2020b) Estimation of regional transition probabilities for spatial dynamic microsimulations from survey data lacking in regional detail. Comput Stat Data Anal. https://doi.org/ 10.1016/j.csda.2020.107048

Chin SF, Harding A (2006) Regional dimensions: creating synthetic small-area microdata and spatial microsimulation models. Technical paper (University of Canberra. National Centre for Social and Economic Modelling), Bd. 33. National Centre for Social and Economic Modelling, Bruce. A.C.T.

Deville JC, Särndal CE (1992) Calibration estimators in survey sampling. J Am Stat Assoc 87(418):376382

Galler HP (1997) Discrete-time and continous-time approaches to dynamic microsimulation reconsidered. Canberra

Goebel J, Grabka MM, Liebig S, Kroh M, Richter D, Schröder C, Schupp J (2018) The German SocioEconomic Panel (SOEP). Jahrb Natl Okon Stat 239(2):345-360. https://doi.org/10.1515/jbnst-20180022

Hannappel M, Troitzsch KG (2015) Mikrosimulationsmodelle. In: Braun N, Saam NJ (Hrsg) Handbuch Modellbildung und Simulation in den Sozialwissenschaften. Springer, Wiesbaden, S 455-490

Hill PB, Kopp J (2013) Familiensoziologie: Grundlagen und theoretische Perspektiven, 5. Aufl. Studienskripten zur Soziologie. Springer VS, Wiesbaden https://doi.org/10.1007/978-3-531-94269-8

van Imhoff E, Post W (1998) Microsimulation methods for population projection. Popul Engl Sel 10(1):97-136

Klemm K, Zorn D (2019) Steigende Schülerzahlen im Primarbereich: Lehrkräftemangel deutlich stärker als von der KMK erwartet. Gütersloh

KMK Kultusministerkonferenz (2018) Vorausberechnung der Schüler- und Absolventenzahlen 2016 bis 2030. Berlin

Kolb JP (2013) Methoden zur Erzeugung synthetischer Simulationsgesamtheiten - Generation of synthetic universes. Universität Trier, Trier (Dissertation)

van Laarhoven PJ, Aarts EHL (1987) Simulated annealing: theory and applications. Mathematics and its applications, Bd. 37. Springer, Dordrecht https://doi.org/10.1007/978-94-015-7744-1

Li J (2011) Dynamic microsimulation for public policy analysis. MGSoG dissertation series / Maastricht Graduate School of Governance, Bd. 24. Boekenplan, Maastricht (zugleich Dissertation: Maastricht University)

Li J, O’Donoghue C (2013) A survey of dynamic microsimulation models: uses, model structure and methodology. Int J Microsimul 6(2):3-55

Manzo G (2007) Variables, mechanisms, and simulations: can the three methods be synthesized? Rev Fr Sociol 48(5):35-71. https://doi.org/10.3917/rfs.485.0035

Ministerium für Bildung, Wissenschaft, Weiterbildung und Kultur (2014) Schulgesetz (SchulG). Mainz

Münnich R, Gabler S, Ganninger M, Burgard JP, Kolb JP (2012) Stichprobenoptimierung und Schätzung im Zensus 2011. Wiesbaden

Münnich R, Burgard JP, Vogt M (2013) Small Area-Statistik: Methoden und Anwendungen. ASTA Wirtsch Sozialstat Arch 6(3-4):149-191

Münnich R, Schnell R, Kopp J, Stein P, Zwick M, Dräger S, Merkle H, Obersneider M, Richter N, Schmaus S (2020) Zur Entwicklung eines kleinräumigen und sektorenübergreifenden Mikrosimulationsmodells für Deutschland. In: Hannappel M, Kopp J (Hrsg) Mikrosimulationen. Springer VS, Wiesbaden, S 109-138 
Münnich R, Schnell R, Brenzel H, Dieckmann H, Dräger S, Emmenegger J, Höcker P, Kopp J, Merkle H, Neufang K, Obersneider M, Reinhold J, Schaller J, Schmaus S, Stein P (2021) A population based regional dynamic microsimulation of Germany: the mikrosim model. Methods Data Anal $15(2): 241-264$

Neutze M (2015) Gitterbasierte Auswertungen des Zensus 2011. Stadtforsch Stat 2:64-67

OpenStreetMap contributors (2021) Data and maptiles. https://www.openstreetmap.org (https://www. openstreetmap.org/copyright, Data is published under the Open Database License (ODbL) 1.0). Zugegriffen: 9. Feb. 2022

Orcutt GH (1957) A new type of socio-economic system. Rev Econ Stat 39(2):116-123

Ottenbacher M (2018) TILL kompakt 2018: Kommentierter Auszug aus der Bildungsmonitoring-Datenbank TILL - ,Trierer Informaonssystem Lebenslanges Lernen“ mit dem Schwerpunkt Zuwanderung und Migration. Trier

Rendtel U, Ruhanen M (2018) Die Konstruktion von Dienstleistungskarten mit Open Data am Beispiel des lokalen Bedarfs an Kinderbetreuung in Berlin. AStA Wirtsch Sozialstat Arch 12(3-4):271-284. https://doi.org/10.1007/s11943-018-0235-y

Rendtel U, Neudecker A, Fuchs L (2021) Ein neues Web-basiertes Verfahren zur Darstellung der Coronainzidenzen in Raum und Zeit. Asta Wirtsch Sozialstat Arch 15(2):93-106

Saam NJ (2015) Simulation in den Sozialwissenschaften. In: Braun N, Saam NJ (Hrsg) Handbuch Modellbildung und Simulation in den Sozialwissenschaften. Springer, Wiesbaden, S 61-95

Statistische Ämter des Bundes und der Länder (2014) Zensus 2011: Zensus Kompakt. Endgültige Ergebnisse. Stuttgart

Statistisches Bundesamt (2017) Datenhandbuch zum Mikrozensus Scientific Use File 2012. https://www. forschungsdatenzentrum.de/sites/default/files/mz_2012_suf_dhb.pdf. Zugegriffen: 9. Feb. 2022

Statistisches Bundesamt (2018) Schulen auf einen Blick: Ausgabe 2018. Wiesbaden

Statistisches Bundesamt (2019) Bevölkerung im Wandel. Annahmen und Ergebnisse der 14. koordinierten Bevölkerungsvorausberechnung. Wiesbaden

Tanton R (2014) A review of spatial microsimulation methods. Int J Microsimul 7(1):4-25

Tanton R (2018) Spatial microsimulation: developments and potential future directions. Int J Microsimul 11(1):143-161

Zinn S (2012) A mate-matching algorithm for continous-time microsimulation models. Int J Microsimul $5(1): 31-51$

Hinweis des Verlags Der Verlag bleibt in Hinblick auf geografische Zuordnungen und Gebietsbezeichnungen in veröffentlichten Karten und Institutsadressen neutral. 